\title{
Morphology parameters: substructure identification in X-ray galaxy clusters ${ }^{\star}$
}

\author{
Viral Parekh ${ }^{1,2}$, Kurt van der Heyden ${ }^{1}$, Chiara Ferrari ${ }^{3}$, Garry Angus ${ }^{1,4}$, and Benne Holwerda ${ }^{5}$ \\ 1 Astrophysics, Cosmology and Gravity Centre (ACGC), Astronomy Department, University of Cape Town, \\ Private Bag X3, 7700 Rondebosch, Republic of South Africa \\ e-mail: viral . parekh2912@gmail .com \\ 2 Raman Research Institute, Sadashivanagar, 560080 Bangalore, India \\ 3 Laboratoire Lagrange, UMR7293, Université de Nice Sophia-Antipolis, CNRS, Observatoire de la Côte d' Azur, \\ 06300 Nice, France \\ ${ }^{4}$ Department of Physics and Astrophysics, Vrije Universiteit Brussel, Pleinlaan 2, 1050 Brussels, Belgium \\ 5 European Space Agency (ESTEC), Keplerlaan 1, 2200 AG Noordwijk, The Netherland
}

Received 3 May 2014 / Accepted 21 November 2014

\begin{abstract}
Context. In recent years multi-wavelength observations have shown the presence of substructures related to merging events in a large proportion of galaxy clusters. Clusters can be roughly grouped into two categories - relaxed and non-relaxed - and a proper characterisation of the dynamical state of these systems is crucial for both astrophysical and cosmological studies.

Aims. In this paper we investigate the use of a number of morphological parameters (Gini, $M_{20}$, concentration, asymmetry, smoothness, ellipticity, and Gini of the second-order moment, $G_{M}$ ) introduced to automatically classify clusters as relaxed or dynamically disturbed systems.

Methods. We apply our method to a sample of clusters at different redshifts extracted from the Chandra archive and investigate possible correlations between morphological parameters and other X-ray gas properties.

Results. We conclude that a combination of the adopted parameters is a very useful tool for properly characterising the X-ray cluster morphology. According to our results, three parameters - Gini, $M_{20}$, and concentration - are very promising for identifying cluster mergers. The Gini coefficient is a particularly powerful tool, especially at high redshift, because it is independent of the choice of the position of the cluster centre. We find that high Gini $(>0.65)$, high concentration $(>1.55)$, and low $M_{20}(<-2.0)$ values are associated with relaxed clusters, while low Gini $(<0.4)$, low concentration $(<1.0)$, and high $M_{20}(>-1.4)$ characterise dynamically perturbed systems. We also estimate the X-ray cluster morphological parameters in the case of radio loud clusters. Since they are in excellent agreement with previous analyses we confirm that diffuse intracluster radio sources are associated with major mergers.
\end{abstract}

Key words. galaxies: clusters: intracluster medium $-\mathrm{X}$-rays: galaxies: clusters

\section{Introduction}

It is now well proven that massive galaxy clusters form and evolve at the intersection of cosmic web filaments through merging and accretion of lower mass systems (e.g. Maurogordato et al. 2011, and references therein). Huge gravitational energy is released during cluster collisions $\left(\approx 10^{64} \mathrm{erg}\right)$, and several billion years are required for the cluster to re-establish a situation of (quasi-)equilibrium after a major merger episode.

Both observations and numerical simulations have shown that merging events deeply affect the properties of the different cluster components (e.g. Ferrari et al. 2008, and references therein). Multiple merger episodes could, for instance, be responsible for disturbing the dynamically relaxed cores of the X-ray emitting hot intracluster medium (ICM, e.g. Burns et al. 2008), as well as the ICM density, temperature and metallicity distribution (e.g. Kapferer et al. 2006). In addition, it is well known that a disturbed X-ray morphology is typical of dynamically perturbed galaxy clusters (Kapferer et al. 2006, and references therein). The presence of substructures, a highly elliptical cluster X-ray morphology, or an X-ray centroid variation

\footnotetext{
* Appendix A is available in electronic form at http://www. aanda.org
}

are typical features that suggest that a cluster is not virialized. This has important implications both for using clusters as tools for cosmology, and for studying the complex gravitational and non-gravitational processes acting during large-scale structure formation and evolution, since in both cases we need to know whether observed clusters are relaxed or not.

Joint X-ray and optical studies can provide detailed information about the dynamical state of a cluster (e.g. Ferrari et al. 2006), but they are extremely time demanding from the observational and analysis points of view. For statistical studies of large cluster samples, we need to identify robust indicators that can somehow quantify the cluster dynamical state. Since the morphology of clusters is deeply related to their evolutionary history, different morphological estimators have been proposed. Jones \& Forman (1992) classified galaxy clusters observed by the Einstein X-ray satellite into six morphological classes that include single, elliptical, offset centre, primary with small secondary, bimodal, and complex. Several studies have tried to quantify the fraction of dynamically disturbed clusters from morphological analyses. Jones \& Forman (1999) showed that around $30 \%$ of clusters observed with the Einstein satellite contain substructure. More recently, Schuecker (2005) have noticed in ROSAT observations that $\sim 50 \%$ of clusters have substructure. 
With high resolution telescopes, such as Chandra and XMM, it has become easier to identify subclusters, bimodality, and X-ray centroid shifts in clusters. Some mergers are too complex, however, to be identified only from X-ray morphological analysis and, particularly in the case of high redshift clusters, some special techniques and statistics are required that can provide a more quantitative and robust measure of the degree of the cluster disturbance.

Various techniques have been suggested to provide a more quantitative and qualitative measure of the degree of the cluster disturbance. Power ratios (Buote \& Tsai 1995b; Jeltema et al. 2005; Böhringer et al. 2010) and the emission centroid shift (Mohr et al. 1993; Böhringer et al. 2010) are most commonly used to classify X-ray galaxy clusters from the morphological point of view. Recently, Andrade-Santos et al. (2012) have used a residual flux method; in order to calculate the substructure level in a given X-ray galaxy cluster, they take into account the ratio between number of counts on the residual (which they obtain by subtracting a surface brightness model from the original X-ray image) and on the original cluster images. Weißmann et al. (2013) proposed to use the maximum of the third-order power ratios calculated in annuli of fixed width and constantly increasing radius to measure the degree of substructure. Rasia et al. (2013) have used six different morphology parameters asymmetry, fluctuation of the X-ray brightness (smoothness), hardness ratios, concentration, the centroid-shift method, and third-order power ratio - to characterise simulated clusters. They took hydrodynamical simulation of 60 clusters and passed it through a Chandra telescope simulator with uniform exposure time $(100 \mathrm{ks})$ for all clusters. Out of all of these parameters, they found that only the asymmetry and concentration parameters could straightforwardly and clearly separate relaxed and non-relaxed systems. The smoothness parameter is affected by the choice of radii and smoothing kernel size. The centroid-shift parameter also works reasonably well and leaves only a few overlapping relaxed and non-relaxed clusters. The third- order power ratio technique also depends on the choice of radius and is limited to only detecting substructure near to the cluster centre. More recently Nurgaliev et al. (2013) have used photon asymmetry and central concentration parameters to quantify morphology of high- $z$ clusters that suffer from low photon counts.

In this paper, we investigate seven morphology parameters, which are typically used for galaxy classification, to study $\mathrm{X}$-ray galaxy cluster morphology, and we find which parameters are optimal for identifying substructure or characterising dynamical states. The combination of morphology parameters has been successfully used to classify different galaxy morphologies (Zamojski et al. 2007; Scarlata et al. 2007; Holwerda et al. 2011a), so we want to investigate their usefulness in galaxy cluster classification. Our focus is not limited to separating galaxy clusters into relaxed and non-relaxed categories, but to study correlations between X-ray gas properties and morphology parameters, as well as the evolution of morphological properties of galaxy clusters from the high redshift universe up to the present.

We explore the usefulness of the non-parametric morphology parameters on a subset of the ROSAT $400 \mathrm{deg}^{2}$ cluster sample observed by the Chandra X-ray telescope (Vikhlinin et al. 2009, hereafter V09). We chose this sample because it has good quality $\mathrm{X}$-ray data and also a broad distribution of redshifts. There are in total 85 (49 low $-z(0.02-0.3)$ and 36 high- $z(0.3-0.8)$ ) galaxy clusters in our analysis. In addition, V09 has measured the global properties of the galaxy clusters (such as luminosity, temperature, mass, etc.), which we use to compare with our measured morphology parameters.
As a test case, in this paper, we study the dynamical activity of clusters hosting diffuse radio sources (radio haloes), in particular we focus our attention on clusters taken from Giovannini et al. (2009). Current results suggest a strong link between the presence of diffuse intracluster radio emission and cluster mergers (Ferrari et al. 2008; Feretti et al. 2012, and references therein). Similar to what was done by Cassano et al. (2010b), but using our set of X-ray morphological parameters, in this paper we analyse the X-ray morphology of relaxed clusters and nonrelaxed clusters (which includes both radio quiet and radio loud mergers).

This paper is organised as follows. Section 2 gives a brief introduction to the morphology parameters. Section 3 gives the sample selection and X-ray data reduction. In Sect. 4, we present our results. Section 5 shows the systematic and possible bias on morphology parameters. In Sect. 6, we compare our parameter measures with available cluster global properties. Finally, Sect. 7 presents our discussions and conclusions. We assumed $H_{0}=73 \mathrm{~km} \mathrm{~s}^{-1} \mathrm{Mpc}^{-1}, \Omega_{\mathrm{M}}=0.3$, and $\Omega_{\Lambda}=0.7$ throughout the paper, unless stated otherwise.

\section{Introduction of morphology parameters}

The non-parametric morphology parameters (Gini, $M_{20}$, concentration, asymmetry, smoothness, ellipticity and Gini of the second-order moment) are widely used to automatically separate galaxies of different Hubble types. As an example, they are used for galaxy morphology classification in the analysis of the HST and SDSS galaxy surveys (Abraham et al. 2003; Conselice 2003; Lotz et al. 2004; Zamojski et al. 2007; Holwerda et al. 2011a; Wang et al. 2012).

Abraham et al. (2003), Lotz et al. (2004), and Wang et al. (2012) also revealed the inter-relation between Gini, concentration and $M_{20}$, as well as the possible inter-change between the concentration and Gini parameter for high- $z$ galaxies. This encouraged us to investigate these parameters in more detail in order to characterise the dynamical state of galaxy clusters, particularly at high-z. In this paper we adopt the definition of concentration, asymmetry and smoothness from Conselice (2003), and of the Gini coefficient and $M_{20}$ from Lotz et al. (2004). The Gini of the second-order moment was defined by Holwerda et al. (2011b). The required input parameters for computing the morphological indicators (except for the Gini parameter) are the central position $\left(x_{\mathrm{c}}, y_{\mathrm{c}}\right)$ of the galaxy clusters, as well as a fixed aperture size or area over which these morphology parameters are measured.

We calculate the centre position by first assigning initial coordinates based on visual observation of each cluster image and then allow the flux-weighted coordinates to iterate in a fixed aperture size of $500 \mathrm{kpc}$, for example, until they have converged. The centre coordinates are then the unique point at the centre of the distribution of flux, essentially the light distribution equivalent to the "centre of mass".

\subsection{Gini coefficient and Gini of the second-order moment $\left(G_{M}\right)$}

The Gini parameter is widely used in the field of economics, where it originated as the Lorenz curve (Lorenz 1905). It describes the inequality of wealth in a population. Here we use it as a calculation of flux distribution in a cluster image. If the total flux is equally distributed among the pixels, then the Gini value is equal to naught (there is constant flux across the pixels regardless of whether those pixels are in the projected centre or not); 
but if the total flux is unevenly distributed and belongs to only a small number of pixels, then the Gini value is equal to one. We adopt the following definition from Lotz et al. (2004):

$G=\frac{1}{\bar{K} n(n-1)} \sum_{i}(2 i-n-1) K_{i}$,

where $K_{i}$ is the pixel value in the $i$ th pixel of a given image, $n$ is the total number of pixels in the image, and $\bar{K}$ is the mean pixel value of the image.

We also apply a Gini value to the second-order moment of each pixel, defining Gini of the second-order moment as:

$G_{M}=\frac{1}{\bar{F} n(n-1)} \sum_{i}(2 i-n-1) F_{i}$,

where $F_{i}$ is the second-order moment of each pixel,

$F_{i}=K_{i} \times\left[\left(x-x_{\mathrm{c}}\right)^{2}+\left(y-y_{\mathrm{c}}\right)^{2}\right]$,

where $(x, y)$ is the pixel position with flux value $K_{i}$ in the cluster image, and $\left(x_{\mathrm{c}}, y_{\mathrm{c}}\right)$ is the coordinate of the cluster centre.

\subsection{Moment of light, $M_{20}$}

Lotz et al. (2004) define the total second-order moment $F_{\text {tot }}$ as the flux in each pixel $K_{i}$ multiplied by the squared distance to the centre of the source, summed over all the selected pixels:

$F_{\mathrm{tot}}=\sum_{i} F_{i}=\sum_{i} K_{i}\left[\left(x_{i}-x_{\mathrm{c}}\right)^{2}+\left(y_{i}-y_{\mathrm{c}}\right)^{2}\right]$,

where $\left(x_{\mathrm{c}}, y_{\mathrm{c}}\right)$ is the centre of the cluster.

The second-order moment can be used to trace various properties of galaxy clusters, such as the spatial distribution of multiple bright cores, substructure, or mergers. Here, $M_{20}$ is defined as the normalised second-order moment of the relative contribution of the brightest $20 \%$ of the pixels. To compute $M_{20}$, we rank-order the image pixels by flux, calculate $F_{i}$ over the brightest pixels until their sum equals $20 \%$ of the total selected cluster flux, and then normalise by $F_{\text {tot }}$ :

$M_{20}=\log \left(\frac{\sum_{i} F_{i}}{F_{\text {tot }}}\right)$, while $\sum_{i} K_{i} \leq 0.2 K_{\text {tot }}$,

where $K_{\text {tot }}$ is the total flux of the cluster image (image pixels are selected from the segmentation $\operatorname{map}^{1}$ ), and $K_{i}$ is the flux value for each pixel $i$ (where $K_{1}=$ the brightest pixel, $K_{2}=$ the second brightest pixel, etc.).

\subsection{Concentration, asymmetry and smoothness (CAS)}

Concentration, asymmetry, and smoothness parameters are commonly known as CAS.

Concentration is defined by Bershady et al. (2000) and Conselice (2003) as

$C=5 \times \log \left(\frac{r_{80}}{r_{20}}\right)$,

where $r_{80}$ and $r_{20}$ represent the radius within which $80 \%$ and $20 \%$ of the flux reside, respectively. Concentration is widely used in the classification of cool core, especially among distant

\footnotetext{
1 A map that defines the chosen circular aperture size with all pixels fixed to a value of 1 .
}

clusters. Santos et al. (2008) define the surface brightness concentration parameter for finding cool core clusters at high redshift as:

$c_{\mathrm{sb}}=\frac{C_{r}(r<40 \mathrm{kpc})}{C_{r}(r<400 \mathrm{kpc})}$,

where $C_{r}(r<40 \mathrm{kpc})$ and $C_{r}(r<400 \mathrm{kpc})$ are the integrated surface brightness within $40 \mathrm{kpc}$ and $400 \mathrm{kpc}$, respectively. Instead of physical radii, we use the percentages of total flux within a given aperture size. This has an advantage (atleast in low- $z$ clusters) in that the flux is independent of angular bin size and galaxy cluster redshift. Our sample covers the redshift range $0.02<z<0.9$. This means that, by adopting a pixel size of $2^{\prime \prime}, 250 \mathrm{kpc}$ corresponds to a pixel range from $\sim 16$ to $\sim 225$. To avoid this large deviation in pixel spread, it is best to use various percentages of the total flux of the galaxy clusters. For the inner radii we use $20 \%-50 \%$, and for the outer radii we use $80 \%-90 \%$ of the total flux. For example, we use the $C_{5080}$ concentration parameter, which means $50 \%$ of the flux within the inner radii and $80 \%$ within the outer radii.

The asymmetry value, which will give rotational symmetry around the cluster centre, is calculated when a cluster image is rotated by $180^{\circ}$ around its centre $\left(x_{\mathrm{c}}, y_{\mathrm{c}}\right)$ and is then subtracted from its original image:

$A=\frac{\sum_{i, j}\left|K(i, j)-K_{180}(i, j)\right|}{\sum_{i, j}|K(i, j)|}$,

where $K(i, j)$ is the value of the pixel at the image position $i, j$, and $K_{180}(i, j)$ is the value of the pixel in the cluster's image rotated by $180^{\circ}$ around its centre. The asymmetry value is sensitive to any region of the cluster that is responsible for asymmetric flux distribution. If the substructure affects the flux distribution at any scale, we can therefore pick it up from the asymmetry value for that galaxy cluster.

The smoothness parameter can be used to identify any patchy flux distribution expected in non-relaxed clusters. By smoothing a cluster image with a filter of width $\sigma$, high frequency structures can be removed from the image. At this point the original image is subtracted from this newly smoothed, lower resolution image. The effect is to produce a residual map that has only highfrequency components of the galaxy cluster's flux distribution. The flux of this residual image is then summed and divided by the total flux of the original cluster image in order to find its smoothness value,

$S=\frac{\sum_{i, j}\left|K(i, j)-K_{\mathrm{s}}(i, j)\right|}{\sum_{i, j}|K(i, j)|}$,

where $K_{\mathrm{s}}(i, j)$ is the pixel in a smoothed image. Here we choose a Gaussian smoothing kernel of $\sigma=12^{\prime \prime}$ as an arbitrary scale to smooth the cluster image.

\subsection{Ellipticity}

Ellipticity is commonly defined by the ratio between a semimajor axis $(A)$ and a semi-minor axis $(B)$ as

$E=1-\frac{B}{A}$, 
where $A$ and $B$ can be computed directly from the second-order moments of the flux in the cluster image as:

$$
\begin{aligned}
& A^{2}=\frac{\overline{x^{2}}+\overline{y^{2}}}{2}+\sqrt{\left(\frac{\overline{x^{2}}-\overline{y^{2}}}{2}\right)^{2}+\overline{x y}^{2}} \\
& B^{2}=\frac{\overline{x^{2}}+\overline{y^{2}}}{2}-\sqrt{\left(\frac{\overline{x^{2}}-\overline{y^{2}}}{2}\right)^{2}+\overline{x y}^{2},}
\end{aligned}
$$

where

$$
\begin{aligned}
& \bar{x}=\frac{\sum_{i \in S} K_{i} x_{i}}{\sum_{i \in S} K_{i}}, \quad \bar{y}=\frac{\sum_{i \in S} K_{i} y_{i}}{\sum_{i \in S} K_{i}}, \\
& \overline{x^{2}}=\frac{\sum_{i \in S} K_{i} x_{i}^{2}}{\sum_{i \in S} K_{i}}-\bar{x}^{2}, \\
& \overline{y^{2}}=\frac{\sum_{i \in S} K_{i} y_{i}^{2}}{\sum_{i \in S} K_{i}}-\bar{y}^{2}, \\
& \overline{x y}=\frac{\sum_{i \in S} K_{i} x_{i} y_{i}}{\sum_{i \in S} K_{i}}-\bar{x} \bar{y} .
\end{aligned}
$$

Here, $\left(x_{i}, y_{i}\right)$ is the $(x, y)$ coordinate of the image of a pixel $i$ of value $K_{i}$ inside an area $S$.

\subsection{Uncertainty estimation}

There are three sources of uncertainty in the calculation of the morphology parameters. These are (1) shot noise in the image pixel values; (2) uncertainties in the centre of the cluster; and (3) variation in the area over which morphology parameters are calculated. The first two uncertainties can be approximated using a number of iterations of the Monte Carlo method. For estimating the third uncertainty, we use a jackknifing technique.

The shot noise effect can be approximated by replacing each pixel value with a Poisson random variable of the mean value of each pixel for the given image, and recalculating the parameters a number of times. After a set number of iterations (in our case 10), the rms of the spread in parameter values is an approximation of uncertainty in the parameters.

Resultant uncertainty from variation in the central position of a cluster is computed by deviating the input $\left(x_{\mathrm{c}}, y_{\mathrm{c}}\right)$ coordinate within a fixed Gaussian width $\left(\sim 30^{\prime \prime}\right.$ in our case). We then recalculate the parameters for several $\left(x_{\mathrm{c}}, y_{\mathrm{c}}\right)$ values. After a number of iterations (10) (Holwerda et al. 2011c), we compute the rms of the spread in parameter values as an approximate value of the uncertainty in the parameters.

The Gini coefficient is less sensitive to the shot noise than to changes in area, as the pixels are ordered first and do not depend on the adopted central position of the cluster in any way. We estimated its uncertainty from a shot noise and the rms in Gini values from a series of subsets of the pixels in the image (by allowing the pixels to be varied using Poisson statistics for a given area). This is known as jackknife error estimation.
Yitzhaki (1991) suggested the use of the jackknife approach to estimate an uncertainty in the Gini coefficient. The jackknife and shot noise uncertainty estimates in the Gini coefficient are similar within an order of magnitude.

We used an error propagation formula to combined the shot noise and central position uncertainties for all parameters except the Gini coefficient. For this, we combined the uncertainties from the shot noise and the jackknife estimation. In this work, therefore, all reported uncertainties are a combination of those mentioned above.

\section{Sample selection and data reduction}

\subsection{Chandra sample}

For our analysis, we required high quality data, particularly for high- $z$ clusters. Burenin et al. (2007) compiled a $400 \mathrm{deg}^{2}$ galaxy cluster catalogue based on the ROSAT PSPC survey. They detected a large number of extended X-ray sources with $f>1.4 \times 10^{-13} \mathrm{erg} \mathrm{s}^{-1} \mathrm{~cm}^{-2}$ in the soft $(0.5-2 \mathrm{keV})$ band. They compared all their detections with their optical counterparts and confirmed 266 out of 283 as galaxy clusters. From this catalogue, a subsample of the low (0.02-0.3)- $z$ and high $(0.3-0.9)-z$ galaxy clusters has been observed with the Chandra X-ray telescope (V09). Figure 1a shows the distribution of redshift, while Figure $1 \mathrm{~b}$ shows the luminosity distribution. In this plot, median $z=0.0853$ and median luminosity $=1.8 \times 10^{44}(\mathrm{erg} / \mathrm{s})$ $(0.5-2 \mathrm{keV})$. Tables 1 and 2 list the low- and high- $z$ samples of galaxy clusters, respectively. We normalised $H$ dependent quantities (e.g. $L_{\mathrm{X}}$ and mass) with $H_{0}=73 \mathrm{~km} \mathrm{~s}^{-1} \mathrm{Mpc}^{-1}$.

Properties of each cluster provided by the V09 are useful in comparison with their X-ray morphology. The total X-ray luminosity is calculated over the $0.5-2 \mathrm{keV}$ band from accurate Chandra flux. The average temperature is measured from the spectrum integrated over a $[0.15-1] R_{500}{ }^{2}$ annulus.

In agreement with V09, we used the "cuspiness" parameter defined by Vikhlinin et al. (2007) to distinguish between cooling flow (relaxed) and non-cooling flow (unrelaxed) clusters. Based on this classification, we used 49 (34 relaxed +15 non-relaxed) low- $z$ clusters and 36 (12 relaxed +24 non-relaxed) high- $z$ clusters.

There are three high- $z$ and two low- $z$ clusters that have multiple components in their images:

- There are two subclumps visible in the high redshift $1701+6414$ cluster. We used the N clump in our calculation. The other clump (in the SW) is a foreground low- $z$ X-ray cluster, confirmed by the NED database.

- In the case of the high redshift system $1641+4001$, there is a small clump (foreground galaxy) in the SW, which we excluded from our analysis.

- Double components are visible in the 0328-2140 system. One is in the E and the other in the $\mathrm{W}$, the latter being a low redshift interloper. We used the high redshift E cluster.

- In A85, we focused on only the main $\mathrm{N}$ relaxed cluster, excluding the small S clump (Kempner et al. 2002).

- In A1644 we excluded the small N component, and focused on the main larger S cluster only (Johnson et al. 2010; Reiprich et al. 2004).

${ }^{2} R_{500}$ is the radius defined as enclosing a region with an over-density $\Delta=500$ times the critical density at the cluster redshift. 
V. Parekh et al.: Morphology parameters: substructure identification in X-ray galaxy clusters
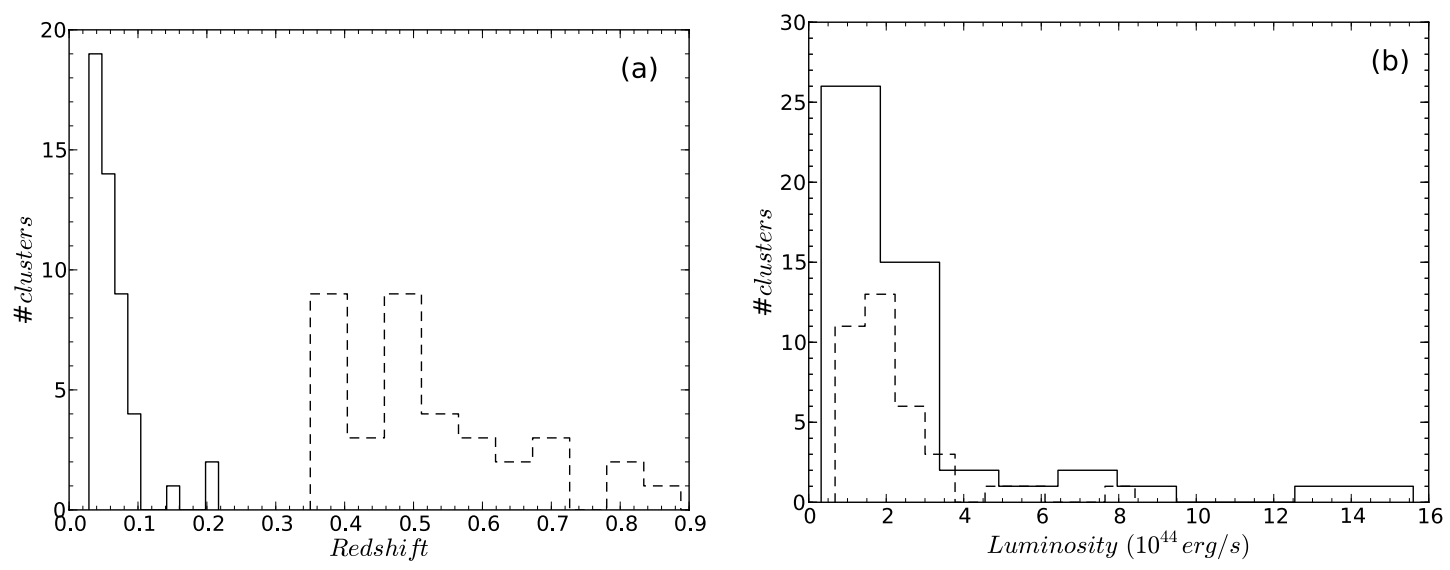

Fig. 1. Redshift (a) and luminosity (b) distribution for low- and high- $z$ clusters (from the V09). Solid line $=$ low- $z$; and dashed line $=$ high- $z$ clusters.

\subsection{Chandra data reduction and image preparation}

In our sample, each cluster had at least 1500-2000 photons (V09), which ensured good signal-to-noise ratio $(\mathrm{S} / \mathrm{N})$. We visually checked all these clusters and verified that there was no cluster peak emission or centroid too close to the CCD edges. There are still a number of cases where cluster emission falls within CCD gaps, and this can potentially influence our results. We divided the counts image by the corresponding exposure map and applied light smoothing (as mentioned below) so as to minimise the effects of CCD gaps. This inspection revealed that the relaxed cluster A2634 (Obs-Id 4816, ACIS-S) alone had low total counts. It was therefore excluded from our sample so we now have a total of 84 clusters in the sample.

We processed all Chandra archival data with CIAO version 4.3 and CALDB version 4.4.6. We first used the chandra_repro task to reprocess all ACIS imaging data. This script creates a new second-level event file, as well as a bad pixel file, by reading the data from the standard Chandra data distribution. After reprocessing, we removed any high background flares ( $3 \sigma$ clipping) with the task lc_sigma_clip routine and then attached the good time interval (GTI) file to the events. All of our event files included the $0.3-7 \mathrm{keV}$ broad energy band. We binned each event file with $\sim 2^{\prime \prime}$ pixels. We detected point sources in observation using the wavdetect $\operatorname{task}^{3}$ with scale $=1$, 2, 4, 8, and 16, which would be a reasonable default for Chandra data. This scale parameter gives the wavelet radii in image pixels. Then a wavelet transform is performed for each scale in the given radii list to estimate source properties. We also supplied information on the size of the PSF at each pixel in the image using energy value of 1.49 . We then selected elliptical background annuli (twice the point source detected region) around each detected point source and removed the point sources using the dmfilth task ${ }^{4}$, which replaces pixel values in selected source regions of an image with values interpolated from the surrounding background region. We used the POISSON interpolation method, which randomly samples values in the selected source regions from the Poisson distribution, whose mean is that of the pixel values in the background region. We took care not to remove a point source that was detected close to the cluster centre, because in most cases, this point source was simply the peak of the surface brightness distribution rather than

\footnotetext{
3 http://cxc.harvard.edu/ciao4.3/threads/wavdetect/

4 http://cxc.harvard.edu/ciao4.3/threads/

diffuseemission/
}

an actual X-ray point source. We also aimed to study the effect of cluster-surrounded point sources on parameters, so we calculated morphology parameters for both images, i.e. one with all point sources and the other without point sources (except central point source). For creating the exposure map, we used the fluximage $\operatorname{task}^{5}$ with broad band energy $(0.3-7 \mathrm{keV})$, then divided the count image by the exposure map to remove the CCD gaps, vignetting, and telescope effects. This task generates the aspect histogram, instrument, and exposure maps automatically. We also used the dmimg thresh task to perform a $5 \%$ total count cut to enable uniform exposure everywhere in the image. Two or more of the available multiple observations were combined to make a single image. Finally we smoothed the cluster images with a $\sigma=10^{\prime \prime}$ Gaussian width. Smoothing is very important in calculating the Gini coefficient, because there will be zero count pixels in the unsmoothed count image, which will not contribute to the flux distribution calculation and will change the Gini value greatly. For calculating the smoothness parameter, we initially used unsmoothed cluster images.

Because of the limited field of view (FOV) of Chandra, in the study of nearby clusters, we decided to use a fixed circular aperture size over which morphology parameters are calculated for individual clusters $(200 \mathrm{kpc}$ for $z<0.05$ and $500 \mathrm{kpc}$ for $z>0.05)$ with sufficient area, and to retain consistency with our morphology parameter calculations on the same relative scale. The possible effects of aperture size are discussed in Sect. 5.2.

\section{Morphology parameter results}

\subsection{Distribution of morphology parameters}

Figure 2 shows the distribution of parameters for our sample of 84 clusters. Except for three parameters, Gini, $M_{20}$, and concentration, most of the parameters show a similar distribution for relaxed and non-relaxed clusters based on the classification described in Sect. 3.1. The smoothness parameter separates the two peaks of relaxed and non-relaxed clusters towards low smoothness and high smoothness, respectively, but there are large numbers of clusters in the overlap region. To further investigate the smoothness parameter, we (1) varied the $\sigma$ value between $0.5^{\prime}$ to $1^{\prime}$ and (2) varied the smoothing size of input cluster images (initial smoothing) on the smoothness parameter (see Sect. 4.4). For the Gini and concentration parameters, non-relaxed clusters are distributed towards low values of Gini and concentration and,

5 http://cxc.harvard.edu/ciao/ahelp/fluximage.html 
Table 1. Low $-z$ (0.02-0.3) cluster sample from the V09.

\begin{tabular}{|c|c|c|c|c|c|c|c|}
\hline Name & $z$ & $\begin{array}{c}\text { Flux } \\
\left(10^{-11} \mathrm{cgs}\right)\end{array}$ & $\begin{array}{l}\text { Luminosity } \\
\left(\mathrm{erg} \mathrm{s}^{-1}\right)\end{array}$ & $\begin{array}{c}\text { Temperature } \\
(\mathrm{keV})\end{array}$ & $\begin{array}{l}\text { Dynamical state } \\
\text { (a) }\end{array}$ & $\begin{array}{l}\text { Dynamical state } \\
\text { (b) }\end{array}$ & $\begin{array}{c}\text { Exposure time } \\
\text { ks }\end{array}$ \\
\hline A3571 & 0.0386 & 7.42 & $2.30 \times 10^{44}$ & $6.81 \pm 0.10$ & $\mathrm{R}$ & $\mathrm{NR}$ & 31.224 \\
\hline A2199 & 0.0304 & 6.43 & $1.23 \times 10^{44}$ & $3.99 \pm 0.10$ & $\mathrm{R}$ & NR & 17.787 \\
\hline 2A 0335 & 0.0346 & 6.24 & $1.55 \times 10^{44}$ & $3.43 \pm 0.10$ & $\mathrm{R}$ & SR & 19.716 \\
\hline A496 & 0.0328 & 5.33 & $1.19 \times 10^{44}$ & $4.12 \pm 0.07$ & $\mathrm{R}$ & NR & 88.878 \\
\hline A3667 & 0.0557 & 4.64 & $3.05 \times 10^{44}$ & $6.33 \pm 0.06$ & M & NR & 60.403 \\
\hline A754 & 0.0542 & 4.35 & $2.70 \times 10^{44}$ & $8.73 \pm 0.00$ & M & SNR & 43.972 \\
\hline A85 & 0.0557 & 4.30 & $2.83 \times 10^{44}$ & $6.45 \pm 0.10$ & $\mathrm{R}$ & NR & 38.201 \\
\hline A2029 & 0.0779 & 4.23 & $5.56 \times 10^{44}$ & $8.22 \pm 0.16$ & $\mathrm{R}$ & SR & 10.739 \\
\hline A478 & 0.0881 & 4.16 & $7.04 \times 10^{44}$ & $7.96 \pm 0.27$ & $\mathrm{R}$ & SR & 42.393 \\
\hline A1795 & 0.0622 & 4.14 & $3.42 \times 10^{44}$ & $6.14 \pm 0.10$ & $\mathrm{R}$ & SR & 91.301 \\
\hline A3558 & 0.0469 & 4.11 & $1.90 \times 10^{44}$ & $4.88 \pm 0.10$ & M & NR & 14.024 \\
\hline A2142 & 0.0904 & 3.94 & $7.00 \times 10^{44}$ & $10.04 \pm 0.26$ & $\mathrm{R}$ & NR & 44.367 \\
\hline A2256 & 0.0581 & 3.61 & $2.58 \times 10^{44}$ & $8.37 \pm 0.24$ & M & SNR & 11.647 \\
\hline A4038 & 0.0288 & 3.48 & $6.01 \times 10^{43}$ & $2.61 \pm 0.05$ & $\mathrm{R}$ & NR & 33.523 \\
\hline A2147 & 0.0355 & 3.47 & $9.14 \times 10^{43}$ & $3.83 \pm 0.12$ & M & SNR & 17.879 \\
\hline A3266 & 0.0602 & 3.39 & $2.61 \times 10^{44}$ & $8.63 \pm 0.18$ & M & SNR & 29.289 \\
\hline A401 & 0.0743 & 3.19 & $3.79 \times 10^{44}$ & $7.72 \pm 0.30$ & $\mathrm{R}$ & NR & 18.005 \\
\hline A2052 & 0.0345 & 2.93 & $7.26 \times 10^{43}$ & $3.03 \pm 0.07$ & $\mathrm{R}$ & NR & 36.754 \\
\hline Hydra-A & 0.0549 & 2.91 & $1.87 \times 10^{44}$ & $3.64 \pm 0.06$ & $\mathrm{R}$ & $\mathrm{R}$ & 89.809 \\
\hline A119 & 0.0445 & 2.47 & $1.03 \times 10^{44}$ & $5.72 \pm 0.00$ & M & NR & 11.537 \\
\hline A2063 & 0.0342 & 2.39 & $5.81 \times 10^{43}$ & $3.57 \pm 0.19$ & $\mathrm{R}$ & NR & 8.777 \\
\hline A1644 & 0.0475 & 2.33 & $1.10 \times 10^{44}$ & $4.61 \pm 0.14$ & M & NR & 18.712 \\
\hline A 3158 & 0.0583 & 2.30 & $1.67 \times 10^{44}$ & $4.67 \pm 0.07$ & $\mathrm{R}$ & NR & 30.921 \\
\hline MKW3s & 0.0453 & 2.08 & $9.02 \times 10^{43}$ & $3.03 \pm 0.05$ & $\mathrm{R}$ & NR & 57.123 \\
\hline A1736 & 0.0449 & 2.04 & $8.69 \times 10^{43}$ & $2.95 \pm 0.09$ & M & SNR & 14.918 \\
\hline EXO0422 & 0.0382 & 2.01 & $6.17 \times 10^{43}$ & $2.84 \pm 0.09$ & $\mathrm{R}$ & $\mathrm{R}$ & 10.001 \\
\hline A4059 & 0.0491 & 2.00 & $1.02 \times 10^{44}$ & $4.25 \pm 0.08$ & $\mathrm{R}$ & NR & 13.236 \\
\hline A3395 & 0.0506 & 1.95 & $1.06 \times 10^{44}$ & $5.10 \pm 0.17$ & M & SNR & 21.094 \\
\hline A2589 & 0.0411 & 1.94 & $6.90 \times 10^{43}$ & $3.17 \pm 0.27$ & $\mathrm{R}$ & NR & 13.478 \\
\hline A 3112 & 0.0759 & 1.89 & $2.36 \times 10^{44}$ & $5.19 \pm 0.21$ & $\mathrm{R}$ & $\mathrm{R}$ & 15.466 \\
\hline A3562 & 0.0489 & 1.84 & $9.32 \times 10^{43}$ & $4.31 \pm 0.12$ & $\mathrm{R}$ & NR & 19.283 \\
\hline A1651 & 0.0853 & 1.80 & $2.85 \times 10^{44}$ & $6.41 \pm 0.25$ & $\mathrm{R}$ & NR & 9.643 \\
\hline A399 & 0.0713 & 1.78 & $1.95 \times 10^{44}$ & $6.49 \pm 0.17$ & M & NR & 48.575 \\
\hline A2204 & 0.1511 & 1.74 & $9.10 \times 10^{44}$ & $8.55 \pm 0.58$ & $\mathrm{R}$ & SR & 9.609 \\
\hline A576 & 0.0401 & 1.72 & $5.82 \times 10^{43}$ & $3.68 \pm 0.11$ & $\mathrm{R}$ & NR & 29.078 \\
\hline A2657 & 0.0402 & 1.62 & $5.50 \times 10^{43}$ & $3.62 \pm 0.15$ & $\mathrm{R}$ & NR & 16.148 \\
\hline A2634 & 0.0305 & 1.61 & $3.11 \times 10^{43}$ & $2.96 \pm 0.09$ & $\mathrm{R}$ & - & 49.528 \\
\hline A3391 & 0.0551 & 1.58 & $1.02 \times 10^{44}$ & $5.39 \pm 0.19$ & $\mathrm{R}$ & NR & 17.461 \\
\hline A2065 & 0.0723 & 1.56 & $1.77 \times 10^{44}$ & $5.44 \pm 0.09$ & M & NR & 49.416 \\
\hline A1650 & 0.0823 & 1.53 & $2.26 \times 10^{44}$ & $5.29 \pm 0.17$ & $\mathrm{R}$ & $\mathrm{R}$ & 27.258 \\
\hline A3822 & 0.0760 & 1.48 & $1.85 \times 10^{44}$ & $5.23 \pm 0.30$ & M & NR & 8.067 \\
\hline S 1101 & 0.0564 & 1.46 & $1.00 \times 10^{44}$ & $2.44 \pm 0.08$ & $\mathrm{R}$ & $\mathrm{R}$ & 9.946 \\
\hline A2163 & 0.2030 & 1.38 & $1.33 \times 10^{45}$ & $14.72 \pm 0.31$ & M & NR & 71.039 \\
\hline ZwCl1215 & 0.0767 & 1.38 & $1.75 \times 10^{44}$ & $6.54 \pm 0.21$ & $\mathrm{R}$ & NR & 11.999 \\
\hline RXJ1504 & 0.2169 & 1.35 & $1.51 \times 10^{45}$ & $9.89 \pm 0.53$ & $\mathrm{R}$ & SR & 13.290 \\
\hline A2597 & 0.0830 & 1.35 & $2.03 \times 10^{44}$ & $3.87 \pm 0.11$ & $\mathrm{R}$ & SR & 26.414 \\
\hline A133 & 0.0569 & 1.35 & $9.33 \times 10^{43}$ & $4.01 \pm 0.11$ & $\mathrm{R}$ & $\mathrm{R}$ & 34.471 \\
\hline A2244 & 0.0989 & 1.34 & $2.89 \times 10^{44}$ & $5.37 \pm 0.12$ & $\mathrm{R}$ & NR & 56.964 \\
\hline A3376 & 0.0455 & 1.31 & $5.72 \times 10^{43}$ & $4.37 \pm 0.13$ & M & NR & 44.267 \\
\hline
\end{tabular}

Notes. (1) Cluster name; (2) redshift; (3) total X-ray flux (0.5-2 keV); (4) total X-ray luminosity (0.5-2 keV); (5) average temperature in [0.15-1] $R_{500}$ annulus; (6) dynamical state (a) (according to the V09); (7) dynamical state (b) (combination of morphology parameters; see Sect. 4.3); $\mathrm{SR}=$ strong relaxed, $\mathrm{R}=$ relaxed, $\mathrm{NR}=$ non-relaxed, $\mathrm{SNR}=$ strong non-relaxed; (8) exposure time.

oppositely, relaxed clusters are characterised by high Gini and concentration values. A similar trend is visible for the $M_{20}$, but the extreme of the relaxed clusters is on the left-hand side (low $M_{20}$ values), and the extreme of the non-relaxed clusters is on the right-hand side (high $M_{20}$ values). Tables A.1 and A.2 list all parameter values, together with $1 \sigma$ uncertainty (Sect. 2.5) for the V09 low- and high-z sample clusters.

We performed the Kolmogorov-Smirnov (K-S) test to investigate whether the relaxed and non relaxed cluster distributions are drawn from the same parent distribution. The results 
V. Parekh et al.: Morphology parameters: substructure identification in X-ray galaxy clusters

Table 2. As in Table 1, but for high-z (0.3-0.9) clusters.

\begin{tabular}{|c|c|c|c|c|c|c|c|}
\hline Name & $z$ & $\begin{array}{c}\text { Flux } \\
\left(10^{-13} \mathrm{cgs}\right)\end{array}$ & $\begin{array}{l}\text { Luminosity } \\
\left(\mathrm{erg} \mathrm{s}^{-1}\right)\end{array}$ & $\begin{array}{c}\text { Temperature } \\
(\mathrm{keV})\end{array}$ & $\begin{array}{l}\text { Dynamical state } \\
\text { (a) }\end{array}$ & $\begin{array}{c}\text { Dynamical state } \\
\text { (b) }\end{array}$ & $\begin{array}{c}\text { Exposure time } \\
\text { ks }\end{array}$ \\
\hline $0302-0423$ & 0.3501 & 15.34 & $5.09 \times 10^{44}$ & $4.78 \pm 0.75$ & $\mathrm{R}$ & SR & 100.41 \\
\hline $1212+2733$ & 0.3533 & 10.53 & $3.51 \times 10^{44}$ & $6.62 \pm 0.89$ & M & NR & 14.581 \\
\hline 0350-3801 & 0.3631 & 1.68 & $6.61 \times 10^{43}$ & $2.45 \pm 0.50$ & M & NR & 23.733 \\
\hline 0318-0302 & 0.3700 & 4.63 & $1.77 \times 10^{44}$ & $4.04 \pm 0.63$ & M & NR & 14.578 \\
\hline $0159+0030$ & 0.3860 & 3.30 & $1.38 \times 10^{44}$ & $4.25 \pm 0.96$ & $\mathrm{R}$ & $\mathrm{R}$ & 19.880 \\
\hline $0958+4702$ & 0.3900 & 2.22 & $1.01 \times 10^{44}$ & $3.57 \pm 0.73$ & $\mathrm{R}$ & $\mathrm{R}$ & 25.231 \\
\hline $0809+2811$ & 0.3990 & 5.40 & $2.43 \times 10^{44}$ & $4.17 \pm 0.73$ & M & SNR & 19.338 \\
\hline $1416+4446$ & 0.4000 & 4.01 & $1.88 \times 10^{44}$ & $3.26 \pm 0.46$ & $\mathrm{R}$ & NR & 29.187 \\
\hline $1312+3900$ & 0.4037 & 2.71 & $1.33 \times 10^{44}$ & $3.72 \pm 1.06$ & M & SNR & 26.421 \\
\hline $1003+3253$ & 0.4161 & 3.04 & $1.48 \times 10^{44}$ & $5.44 \pm 1.40$ & $\mathrm{R}$ & $\mathrm{R}$ & 19.859 \\
\hline 0141-3034 & 0.4423 & 2.06 & $1.28 \times 10^{44}$ & $2.13 \pm 0.38$ & M & SNR & 28.273 \\
\hline $1701+6414$ & 0.4530 & 3.91 & $2.32 \times 10^{44}$ & $4.36 \pm 0.46$ & $\mathrm{R}$ & $\mathrm{R}$ & 49.294 \\
\hline $1641+4001$ & 0.4640 & 1.43 & $9.20 \times 10^{43}$ & $3.31 \pm 0.62$ & $\mathrm{R}$ & NR & 45.345 \\
\hline $0522-3624$ & 0.4720 & 1.47 & $1.01 \times 10^{44}$ & $3.46 \pm 0.48$ & M & NR & 45.999 \\
\hline $1222+2709$ & 0.4720 & 1.39 & $9.61 \times 10^{43}$ & $3.74 \pm 0.61$ & $\mathrm{R}$ & NR & 49.117 \\
\hline $0355-3741$ & 0.4730 & 2.48 & $1.71 \times 10^{44}$ & $4.61 \pm 0.82$ & $\mathrm{R}$ & $\mathrm{R}$ & 27.190 \\
\hline $0853+5759$ & 0.4750 & 1.22 & $8.20 \times 10^{43}$ & $3.42 \pm 0.67$ & M & NR & 42.179 \\
\hline $0333-2456$ & 0.4751 & 1.33 & $9.52 \times 10^{43}$ & $3.16 \pm 0.58$ & M & NR & 34.160 \\
\hline $0926+1242$ & 0.4890 & 2.04 & $1.45 \times 10^{44}$ & $4.74 \pm 0.71$ & M & NR & 18.620 \\
\hline $0030+2618$ & 0.5000 & 2.09 & $1.52 \times 10^{44}$ & $5.63 \pm 1.13$ & M & NR & 57.362 \\
\hline $1002+6858$ & 0.5000 & 2.19 & $1.66 \times 10^{44}$ & $4.04 \pm 0.83$ & M & NR & 19.786 \\
\hline $1524+0957$ & 0.5160 & 2.45 & $2.01 \times 10^{44}$ & $4.23 \pm 0.51$ & M & SNR & 49.886 \\
\hline $1357+6232$ & 0.5250 & 1.90 & $1.58 \times 10^{44}$ & $4.60 \pm 0.69$ & $\mathrm{R}$ & NR & 43.862 \\
\hline $1354-0221$ & 0.5460 & 1.45 & $1.36 \times 10^{44}$ & $3.77 \pm 0.53$ & M & NR & 55.039 \\
\hline $1120+2326$ & 0.5620 & 1.68 & $1.74 \times 10^{44}$ & $3.58 \pm 0.44$ & M & SNR & 70.262 \\
\hline $0956+4107$ & 0.5870 & 1.64 & $1.79 \times 10^{44}$ & $4.40 \pm 0.50$ & $\mathrm{M}$ & NR & 40.165 \\
\hline $0328-2140$ & 0.5901 & 2.09 & $2.23 \times 10^{44}$ & $5.14 \pm 1.47$ & $\mathrm{R}$ & NR & 56.192 \\
\hline $1120+4318$ & 0.6000 & 3.24 & $3.64 \times 10^{44}$ & $4.99 \pm 0.30$ & $\mathrm{R}$ & NR & 19.837 \\
\hline $1334+5031$ & 0.6200 & 1.76 & $2.16 \times 10^{44}$ & $4.31 \pm 0.28$ & $\mathrm{M}$ & NR & 19.492 \\
\hline $0542-4100$ & 0.6420 & 2.21 & $2.83 \times 10^{44}$ & $5.45 \pm 0.77$ & M & NR & 50.008 \\
\hline $1202+5751$ & 0.6775 & 1.34 & $2.15 \times 10^{44}$ & $4.08 \pm 0.72$ & M & SNR & 57.210 \\
\hline $0405-4100$ & 0.6861 & 1.33 & $2.16 \times 10^{44}$ & $3.98 \pm 0.48$ & M & NR & 77.163 \\
\hline $1221+4918$ & 0.7000 & 2.06 & $3.25 \times 10^{44}$ & $6.63 \pm 0.75$ & M & NR & 78.887 \\
\hline $0230+1836$ & 0.7990 & 1.09 & $2.48 \times 10^{44}$ & $5.50 \pm 1.02$ & M & SNR & 67.178 \\
\hline $0152-1358$ & 0.8325 & 2.24 & $5.31 \times 10^{44}$ & $5.40 \pm 0.97$ & $\mathrm{M}$ & NR & 36.285 \\
\hline $1226+3332$ & 0.8880 & 3.27 & $8.19 \times 10^{44}$ & $11.08 \pm 1.39$ & M & NR & 64.21 \\
\hline
\end{tabular}

Table 3. Statistics for the subsample of relaxed (R) and non-relaxed (N-R) clusters, and the combined (C) cluster sample from the V09.

\begin{tabular}{ccccc}
\hline \hline & Mean & Median & K-S probability & R-S probability \\
& R N-R C & R N-R C & & \\
\hline Gini & 0.550 .390 .47 & 0.540 .390 .44 & $1.42 \times 10^{-07}$ & $8.73 \times 10^{-09}$ \\
$M_{20}$ & $-1.96-1.44-1.72$ & $-1.96-1.44-1.73$ & $8.65 \times 10^{-09}$ & $8.63 \times 10^{-11}$ \\
Concentration & 1.380 .951 .18 & 1.330 .921 .11 & $8.87 \times 10^{-08}$ & $2.26 \times 10^{-9}$ \\
Asymmetry & 0.390 .440 .42 & 0.380 .440 .40 & 0.12 & 0.027 \\
Smoothness & 0.681 .050 .85 & 0.611 .090 .94 & 0.0020 & $6.7 \times 10^{-5}$ \\
$G_{M}$ & 0.270 .310 .29 & 0.280 .300 .28 & 0.012 & 0.003 \\
Ellipticity & 0.080 .090 .09 & 0.080 .090 .08 & 0.95 & 0.66 \\
\hline
\end{tabular}

are given for each parameter in Table 3, where a threshold of $1 \%$ means that the probability is $<0.01$, implying a possible rejection of the null hypothesis. In the table we include the Wilcoxon rank sum (R-S) test, which establishes the probability of the two samples with the null hypothesis having the same mean. A threshold of $0.1 \%$ implies that we reject the null hypothesis (that they have the same mean) if the probability is $<0.001$. In addition, the mean and median values for all morphology parameters are supplied. From the statistical tests, we observed a significant separation between the two distributions (relaxed and non-relaxed clusters) for the Gini, $M_{20}$, and concentration, which rejects the null hypotheses that the two samples (relaxed and non-relaxed clusters) are the same and that they have the same mean values. The K-S and R-S probabilities are $<0.01$ and 0.001 , respectively, for the smoothness parameter; but we observed a high incidence of overlapping between relaxed and non-relaxed clusters for the smoothness distribution. We found that the right side peak of non-relaxed clusters (dotted line) in the smoothness distribution corresponds mainly to the high- $z$ clusters that have low S/N compared with nearby clusters (which fall mainly on the left side). Further details concerning the smoothness parameter is given in Sect. 4.4. For the remaining parameters, we cannot reject the 

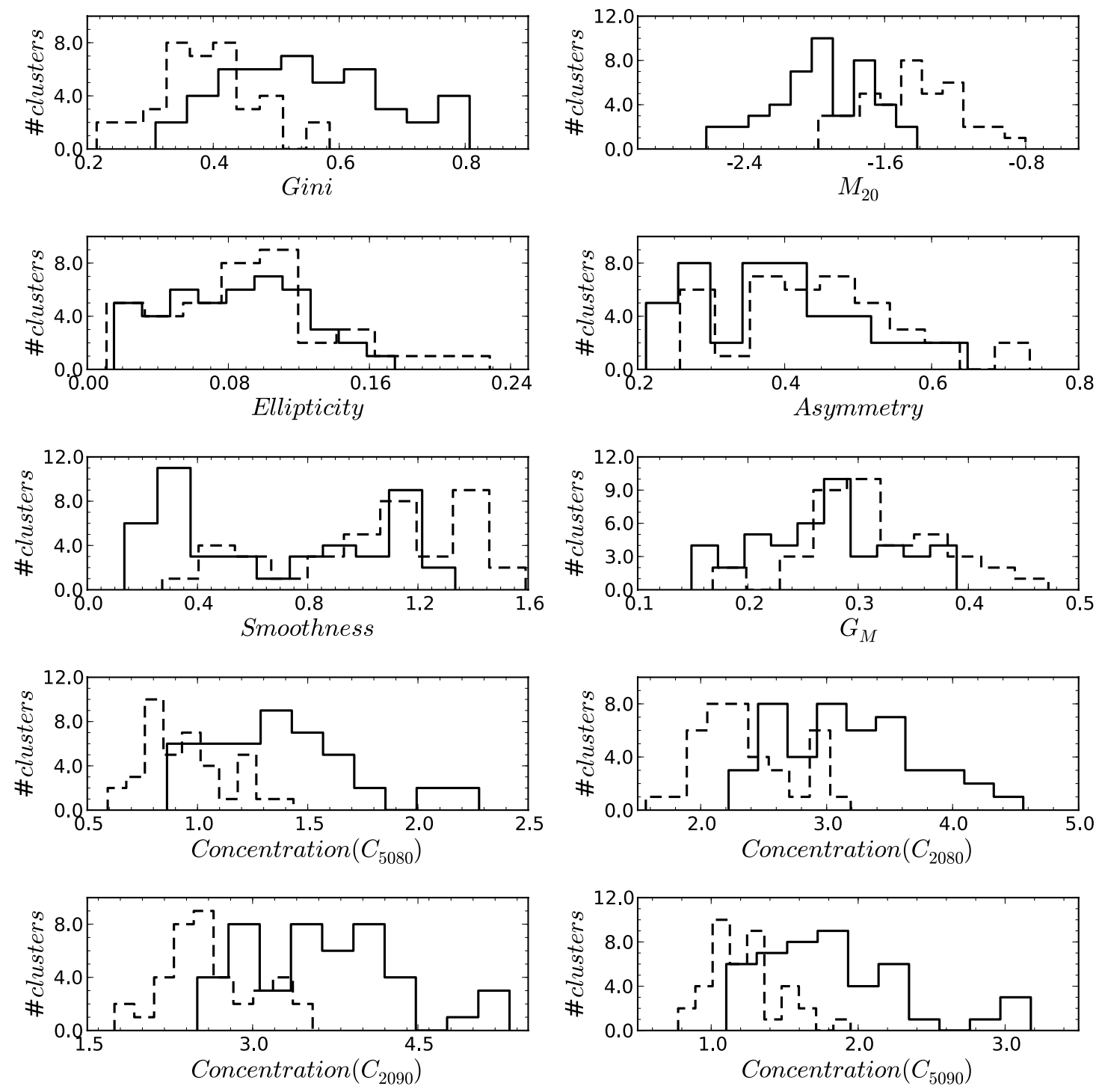

Fig. 2. Seven morphology parameter distributions: solid line = relaxed cluster; dashed line = non-relaxed cluster. All four definitions of the concentration parameter are displayed. Galaxy cluster separation (i.e. relaxed vs. non-relaxed systems) is based on the V09.

above null hypotheses, which means that asymmetry, the Gini of the second-order moment, and ellipticity are not useful in separating relaxed and non-relaxed clusters.

\subsection{Parameter vs. parameter planes}

Using combinations of morphology parameters, we investigated relaxed vs. non-relaxed clusters in the parameter-parameter plane to study the dynamical states of galaxy clusters and the correlation between each morphology parameter. In Fig. 3 we plotted our results in parameter-parameter planes. Three parameters, Gini, concentration, and $M_{20}$, look particularly powerful after combining with other parameters to differentiate between non-relaxed and relaxed clusters. Clusters with different dynamical states (as classified by the V09) occupy distinct regions in the parameter-parameter planes; for example, in the concentration vs. asymmetry plot, all relaxed clusters occupy the upper region, while the lower region is occupied by non-relaxed clusters. In our analysis we did not observe any correlation between cluster dynamical states and ellipticity or asymmetry. As seen in Fig. 3, galaxy clusters within our sample show a range of different morphologies and are not concentrated in particular positions of the parameter-parameter space. This is probably because of the hierarchical cluster formation process, indicating that clusters pass through multiple (merger) phases in their evolution. Each phase is dynamically important and traces the cluster properties. This could help in the understanding of large and complex structure formations in the standard cosmological model.

We used the Spearman rank-order correlation coefficient, $\rho$, to quantify any correlation between parameter pairs. This resulted in a correlation coefficient between ranks of a group of individuals for a given pair of attributes. To calculate $\rho$, it is necessary to assign ranks (low to high or high to low) to a given set of variables, individually. The next step is to measure the deviation between the ranks of variable pairs, square this rank difference, and sum up. The value of the rank-order correlation coefficient varies between -1 and 1 . If the variables are anti-correlated, then $\rho$ falls between -1 and 0 , a value for $\rho$ between 0 and 1 implies a positive correlation between given variables, and $\rho=0$ implies no correlation between variables.

Figure 3 indicates that the concentration is tightly correlated with the Gini, but anti-correlated with the $M_{20}$ (see also 


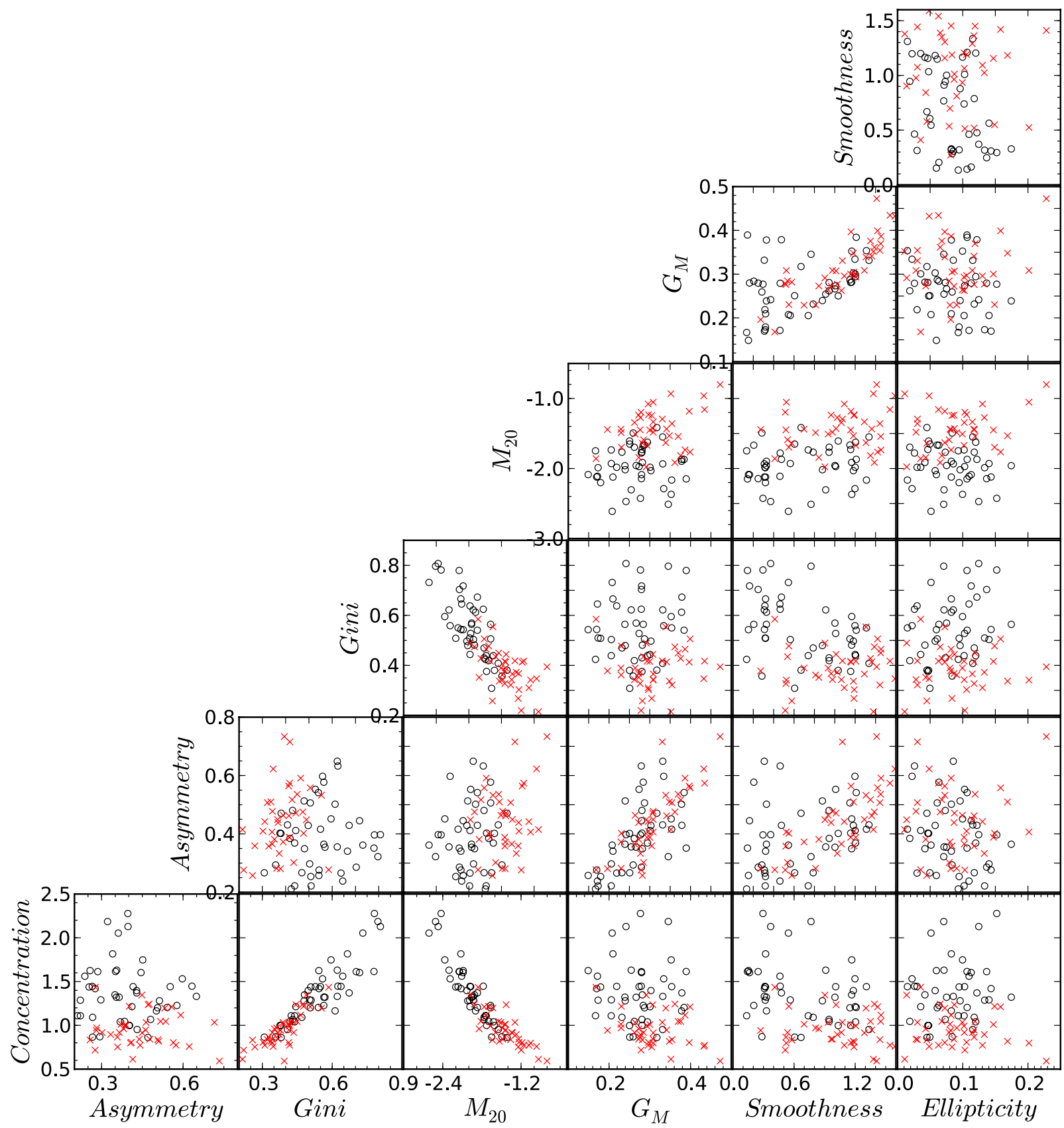

Fig. 3. Seven morphology parameters plotted in the parameter-parameter planes. $C_{5080}$ was plotted as the concentration parameter. $\circ=$ relaxed cluster; $x=$ non-relaxed cluster. Galaxy cluster separation is based on the V09.

Table 4). The concentration (Santos et al. 2008; Hudson et al. 2010; Cassano et al. 2010b) is a useful parameter for separating non-relaxed from relaxed clusters with almost all morphology parameters. Figure 3 illustrates our plot of the $C_{5080}$ concentration parameter. As seen in Fig. 3, relaxed clusters occupy the upper left-hand corner, while non-relaxed clusters occupy the bottom right-hand corner of the $M_{20}$ vs. Gini and concentration planes. In the Gini vs. concentration plane, relaxed clusters fall in the upper right-hand corner, while the bottom left-hand corner is occupied by non-relaxed clusters. In this study we found that the Gini coefficient was also useful in separating non-relaxed clusters from relaxed clusters when plotted against most other parameters. The Gini, concentration, and $M_{20}$ parameters are all inter-related as well (Table 4), and the Gini coefficient could be useful as a proxy of the concentration for detecting substructure in high- $z$ clusters. The advantage of using the Gini coefficient is that it is independent of the precise location of a galaxy cluster's centre. The asymmetry parameter is correlated with Gini of the second-order moment and smoothness parameters (Okabe et al. 2010; Zhang et al. 2010; Rasia et al. 2013). We did not observe any correlation between the other six parameters and ellipticity. In general, three parameters - Gini, $M_{20}$, and concentration are very promising tools in the morphological classification of clusters.

Despite this reasonable division between relaxed and nonrelaxed clusters, we observed an overlap between some clusters, such as A401, A3571, A1651, A3158, A3562, A576, A2063, ZwCl1215, A2657, A2589, A3391, 0355-3741, 1641+4001, $1120+4318,1222+2709,0328-2140$, and $1357+6232$. These are identified as relaxed clusters in the V09, but in our parameter 
space they fall into the non-relaxed region. A401 (Murgia et al. 2010) and A3562 (Venturi et al. 2003) host radio haloes (indicating a possible signature of merger, see Sect. 6.4), which are clearly identified as non-relaxed clusters by our parameters (although they are classified as relaxed in the V09). Dupke et al. (2007) predict a line-of-sight bullet-like cluster in A576, using a combination of Chandra and XMM observations. This gave us confidence that our parameter set provided a good indication of cluster dynamical states. The remaining clusters may be weak mergers (pre- or post-merger) or in intermediate state.

In cluster A85 (Kempner et al. 2002), in addition to the main cool core cluster, two subclusters are visible, in the far $\mathrm{S}$ and NW, respectively. We calculated parameters for the main relaxed cluster and found that it falls into the relaxed category, although very close to the boundary of the non-relaxed side, which could indicate a weak interaction between the subcluster and the main cool core cluster. Based on our measurements, we categorised most relaxed clusters by concentration $>1.55$ and $M_{20}<-2.0$, while intermediate clusters were categorised by $1.0<$ concentration $<1.55$ and $-2.0<M_{20}<-1.4$, and distorted and non-relaxed clusters were categorised by concentration $<1.0$ and $M_{20}>-1.4$. According to the Gini coefficient, most relaxed clusters have a Gini value $>0.65$ and non-relaxed have a Gini value $<0.40$. We chose these boundaries based on visual identification of each cluster morphology (Fig. 3). Mean and median values (Table 3 ) produce a significant overlap due to the presence of a large number of intermediate clusters in our sample.

\subsection{Combination of morphology parameters}

We decided to classify dynamical states of each cluster further based on the combination of Gini, $M_{20}$, and concentration. In Sect. 4.2, we defined three parameter boundaries. Based on the combination of these three parameters, we categorised the V09 sample of clusters into four stages - strong relaxed, relaxed, nonrelaxed, and strong non-relaxed clusters. We selected the following criteria for identifying the dynamical state of each cluster:

- If the Gini, $M_{20}$, and concentration all indicate relaxed state, then the cluster will be "strong relaxed".

- The intermediate state of clusters is further divided into two classes: if one or two parameters fall into the intermediate state and another is relaxed (or non-relaxed), then the cluster will be "relaxed (or non-relaxed)".

- If all three parameters indicate a non-relaxed state, then the cluster will be "strong non-relaxed".

Based on these categories, in the entire V09 cluster sample, $8(\sim 10 \%)$ clusters were strong relaxed, $11(\sim 13 \%)$ were relaxed, $52(\sim 62 \%)$ were non-relaxed, and $13(\sim 15 \%)$ were strong non-relaxed. In the low- $z$ sample, there were $7(\sim 15 \%)$ strong relaxed, $6(\sim 12.5 \%)$ relaxed, $29(\sim 60 \%)$ non-relaxed, and $6(\sim 12.5 \%)$ strong non-relaxed clusters. In the high- $z$ sample, there were $1(\sim 3 \%)$ strong relaxed, $5(\sim 14 \%)$ relaxed, 23 ( $\sim 64 \%)$ non-relaxed, and $7(\sim 19 \%)$ strong non-relaxed clusters. This could suggest that the largest number of clusters (in the V09 sample) are evolving or showing some substructure activity (particularly in the high- $z$ sample) and that fewer clusters are fully evolved. Figure 4 shows the 3D plot of the Gini, $M_{20}$, and concentration parameters. A combination of these three parameters classified galaxy clusters as strong relaxed $(\bullet)$, relaxed $(\diamond)$, non-relaxed $(+)$ and strong non-relaxed clusters $(\times)$. 
V. Parekh et al.: Morphology parameters: substructure identification in X-ray galaxy clusters

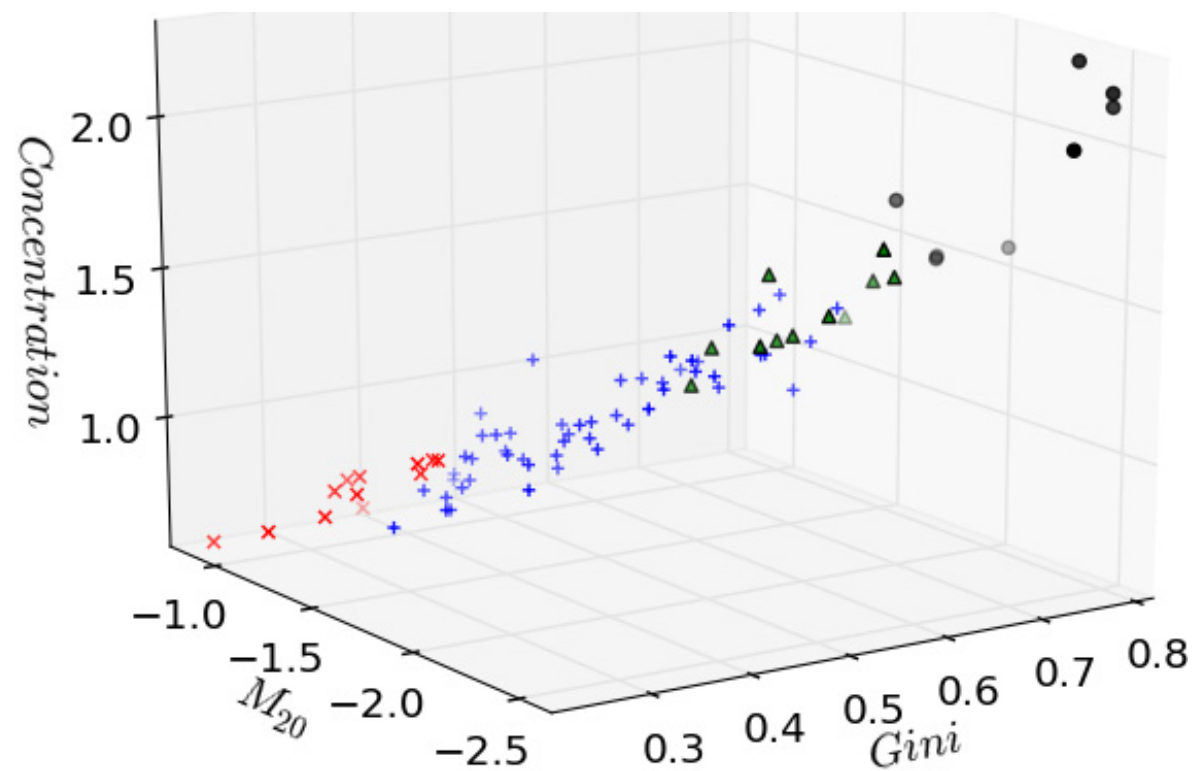

Fig. 4. Gini, $M_{20}$, and concentration parameters plotted in the 3D plot. We plotted $C_{5080}$ as the concentration parameter. $\bullet=$ strong relaxed clusters, $\Delta=$ relaxed clusters, $+=$ non-relaxed clusters, and $\times=$ strong non-relaxed clusters.
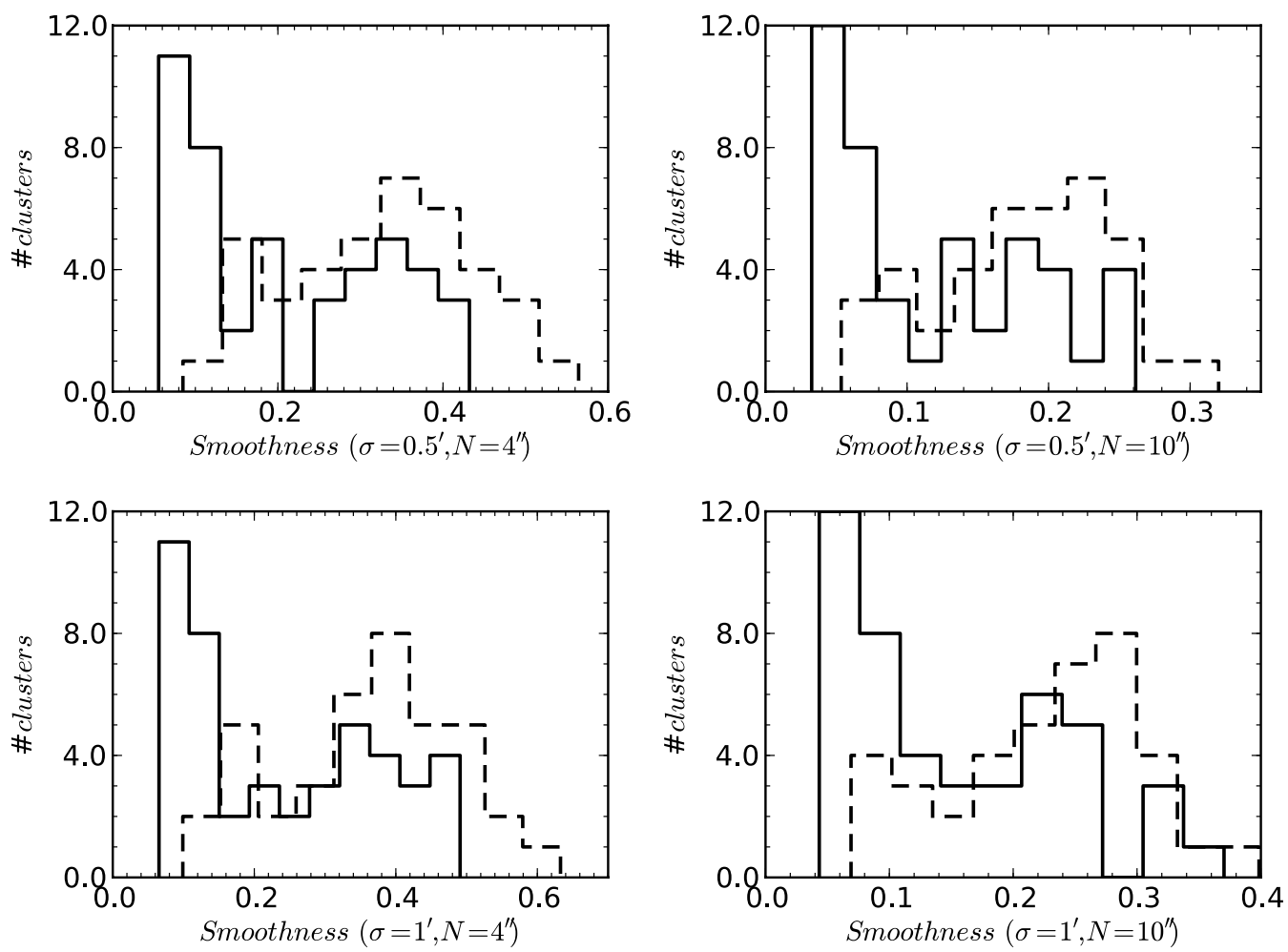

Fig. 5. Calculation of the smoothness parameter for two different Gaussian kernel sizes of fixed angular scale: solid line = relaxed cluster; dashed line $=$ non-relaxed cluster. $N$ shows smoothing size for input cluster image. Galaxy cluster separation is based on the V09.

\subsection{Smoothness and asymmetry parameters}

In this section we test different possible smoothing and angular size issues relating to the smoothness and asymmetry parameters. Okabe et al. (2010) used a $2^{\prime}$ smoothing scale for calculating the fluctuation parameter. They, however, used it for $R_{500}$ radius and the data of XMM-Newton that has a large FOV compared with Chandra. We used $0.5^{\prime}$ (15 pix) and $1^{\prime}$ (30 pix) smoothing scales, $\sigma$, with initial $4^{\prime \prime}$ (2 pix) and $10^{\prime \prime}$ (5 pix) smoothing of input cluster images (which is sufficient to not wash out any underlying substructure features). Results are shown in Fig. 5. We find that the different smoothing scales $(\sigma)$ had little affect on the smoothness parameter. In this analysis, we used a fixed angular size of $2^{\prime \prime} /$ pix to bin each cluster image and the same angular smoothing scale $(\sigma)$ for low- and high- $z$ clusters. This angular scaling could affect the smoothness parameter. To overcome this, and instead of using a fixed angular size, we scaled each cluster image (of low- and high-z) in 

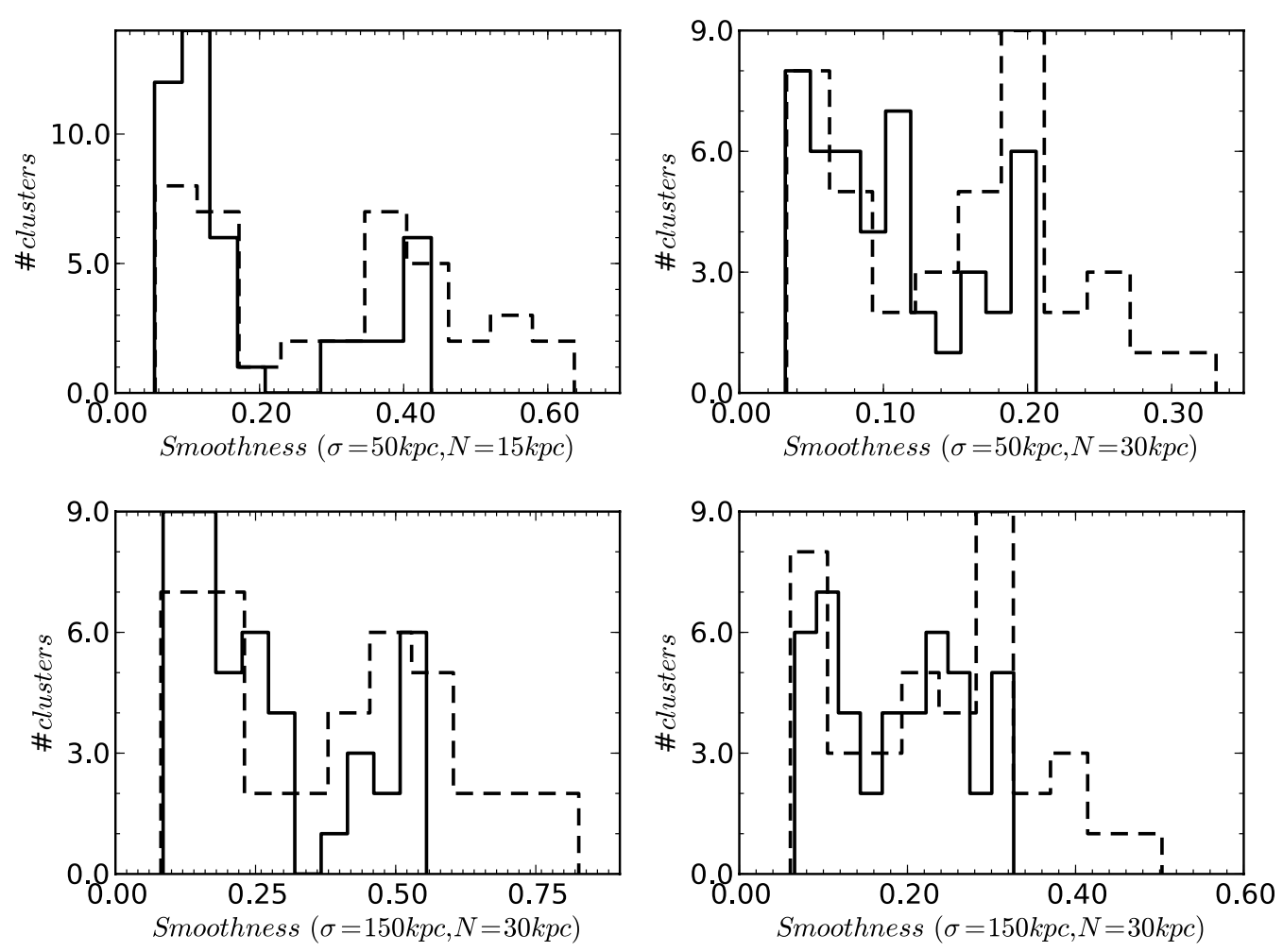

Fig. 6. Calculation of the smoothness parameter for two different Gaussian kernel sizes of fixed physical scale: solid line = relaxed cluster; dashed line $=$ non-relaxed cluster. $N$ shows smoothing size for input cluster image. Galaxy cluster separation is based on the V09.

terms of fixed physical size of $10 \mathrm{kpc} /$ pix. We then used 50 and $150 \mathrm{kpc}$ smoothing scales $(\sigma)$ to smooth cluster images in order to calculate the smoothness parameter. Both of these $\sigma$ values were chosen based on the available aperture size (segmentation map) of cluster for which we calculated the smoothness parameter. We also smoothed cluster input images with (1) 15 (1.5 pix) and (2) 30 (3 pix) kpc Gaussian kernel sizes (which is sufficient for not washing out any underlying substructure features). Figure 6 gives the calculated smoothness parameter for fixed physical smoothing kernel size. The smoothness parameter had barely changed from the previous results, and had still not separated the two classes of cluster, i.e. relaxed and nonrelaxed. Furthermore, most clusters (mainly high- $z$ ) do not have $\gg 1$ count in each (binned) pixel, so, in general, the smoothness is not a good parameter for a large number of clusters in which each has a different exposure time. In Sect. 5.3, we go on to show that the smoothness parameter depends strongly on $\mathrm{S} / \mathrm{N}$.

We also investigated the asymmetry parameter using various smoothing Gaussian kernel sizes to smooth cluster input images. We rotated this smoothed image by $180^{\circ}$, subtracted it from the input image, and normalised it. Our results for the fixed angular and physical scale cluster images are given in Fig. 7. None of the results indicates the separation of relaxed and non-relaxed clusters. As for the smoothness parameter, asymmetry parameter is not useful because the $\mathrm{S} / \mathrm{N}$ is inadequate in a given large sample of clusters (see Sect. 5.3).

\section{Systematics}

We investigated a number of possible systematic effects, discussed below, to study how robust these parameters are in various conditions.

\subsection{Effect of point sources}

To test the effect of point sources detected around a cluster on the calculation of parameters, we calculated morphology parameters on (exposure-corrected and smoothed) cluster images without removing any of the detected point source. We noticed that parameters are fairly robust against the inclusion (or exclusion) of point sources into the parameter calculation. Figure 8 indicates how we plotted the offset for four parameters (Gini, $M_{20}$, concentration, and smoothness) calculated with and without point sources. As we observed, less offset between parameters (with or without point sources) suggests that they are quite robust. The Gini and $M_{20}$, however, each show a small extended tail on the right-hand side (Fig. 8). This result is obvious, because the Gini coefficient includes bright pixels from point sources in the calculation, and thus increases by a low value. In the case of the $M_{20}$, if the point sources are very close to the centre, its value will decrease by a small factor.

\subsection{Aperture size effect}

Aperture radii have an important effect when measuring morphology parameters. We chose to use a fixed physical size radius rather than a fixed overdensity $\left(R_{500}\right)$ radius, because it is difficult to measure $R_{500}$ accurately for non-relaxed clusters. Although a study of the possible bias effects of this choice goes beyond the purpose of this paper, we note that possible bias effects could be introduced when comparing clusters at the same redshift, but characterised by different luminosities (masses). The large variation in aperture size does affect the parameters because they are related to surface brightness. To check the effect of various aperture radii on the parameters, we chose a subsample that included both distant and nearby clusters and calculated 
V. Parekh et al.: Morphology parameters: substructure identification in X-ray galaxy clusters
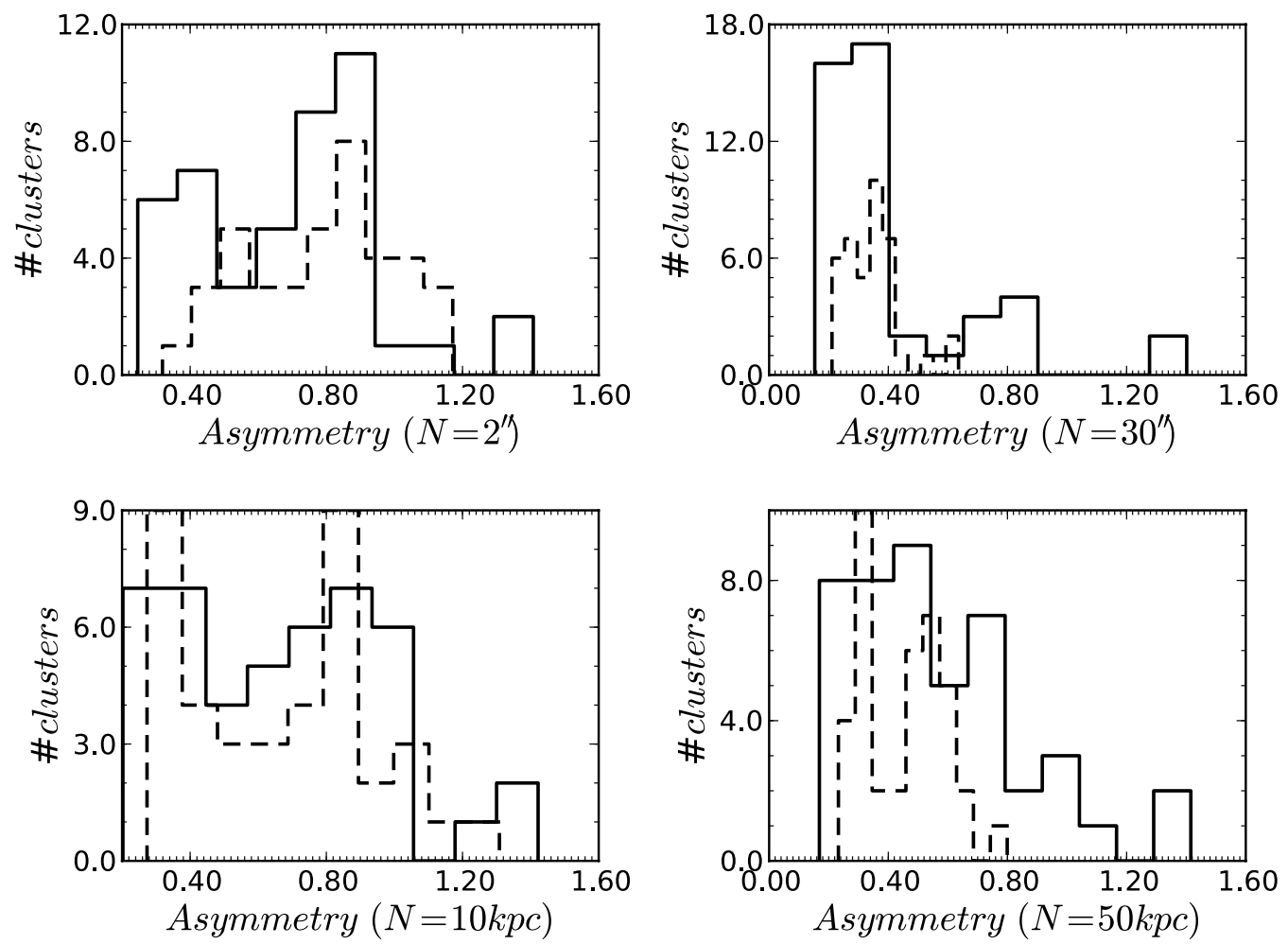

Fig. 7. Calculation of the asymmetry parameter for two different Gaussian kernel sizes of fixed angular and physical scale: solid line $=$ relaxed cluster; dashed line $=$ non-relaxed cluster. $N$ shows smoothing size for input cluster image. Galaxy cluster separation is based on the V09.
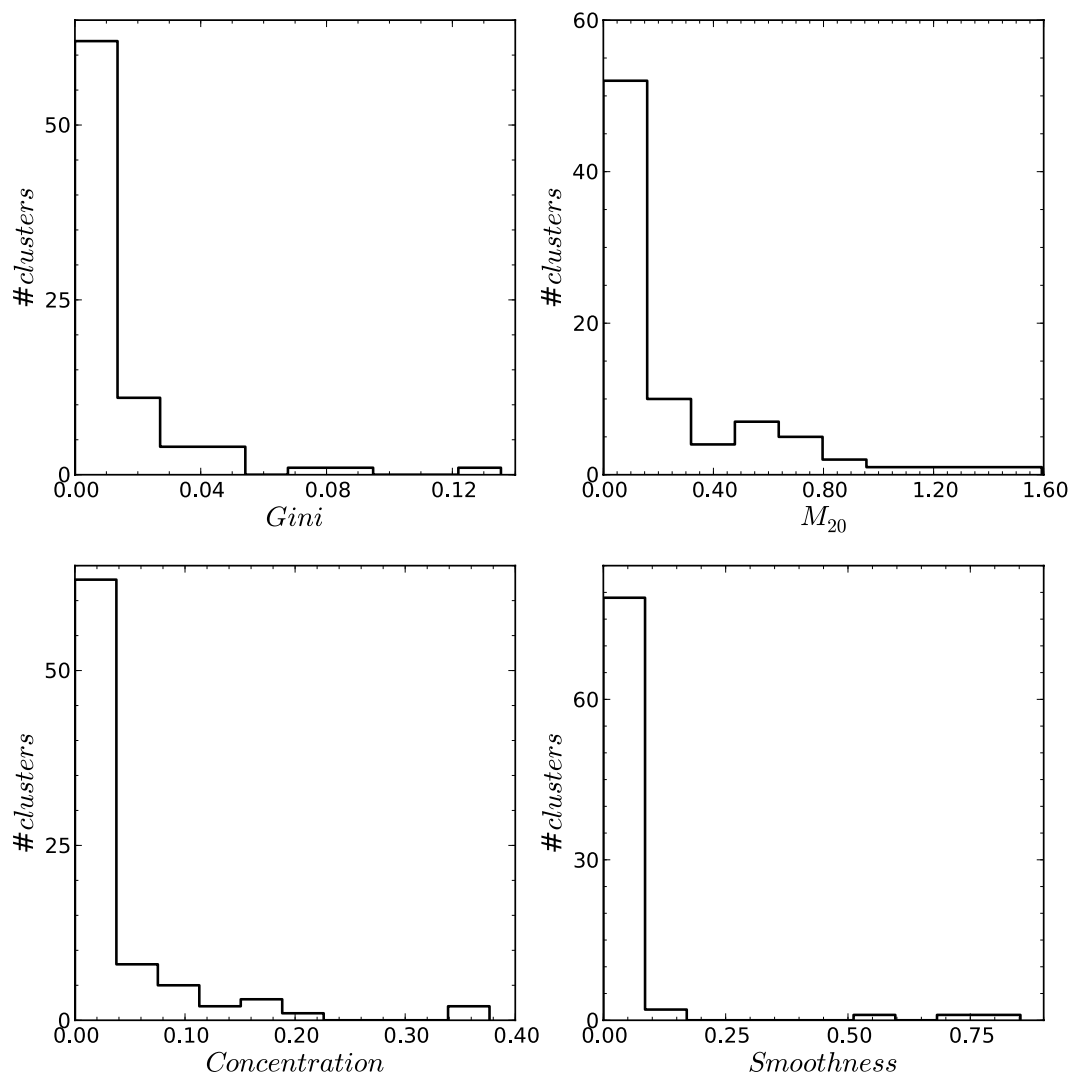

Fig. 8. Four parameters calculated with and without point sources, with plotted offset between them. Here we plotted $C_{5080}$ as the concentration parameter. Less offset suggests that parameters are quite robust. 

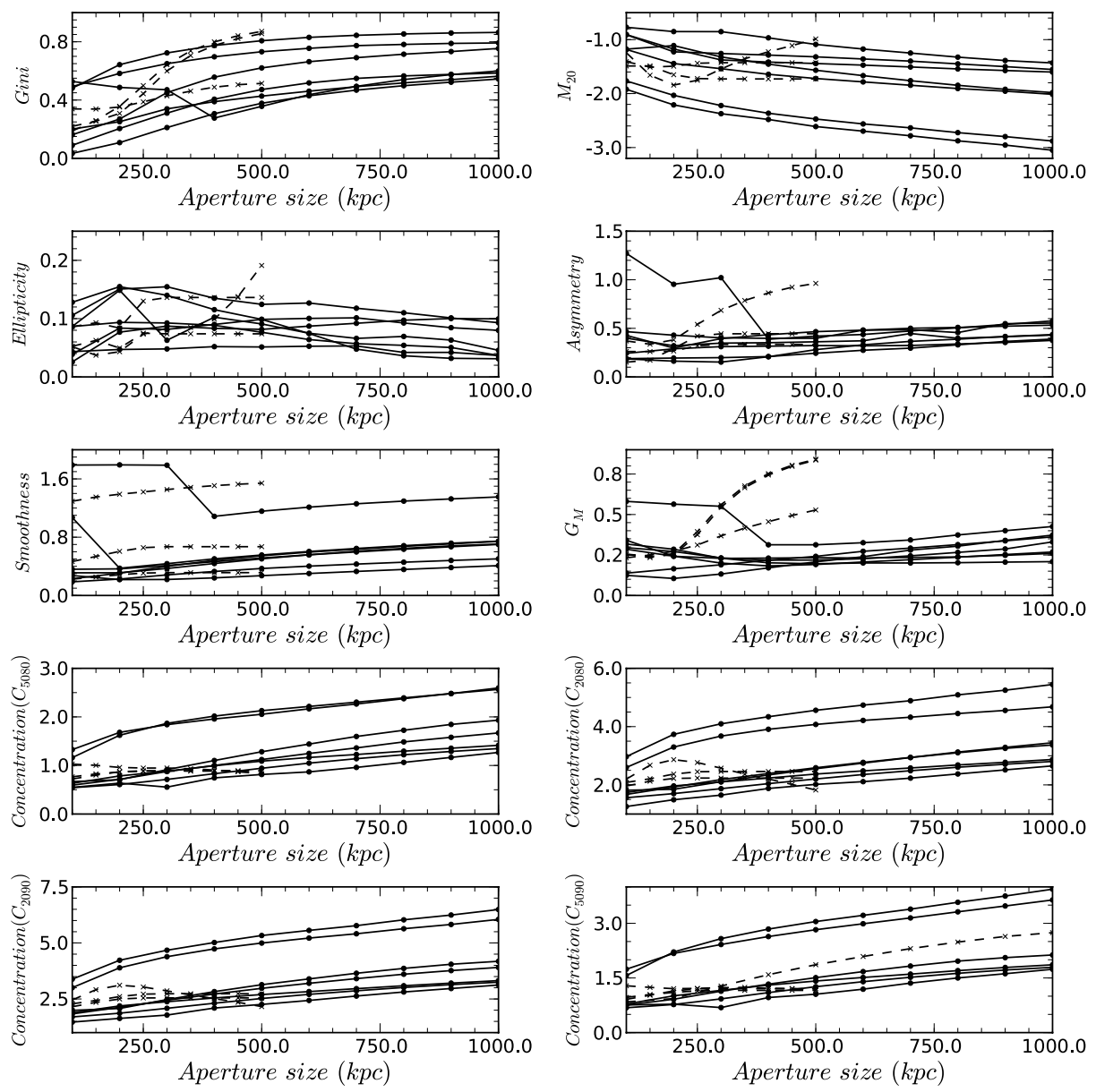

Fig. 9. Morphology parameters calculated for a range of aperture sizes from $100 \mathrm{kpc}$ to $1 \mathrm{Mpc}$ for distant clusters. Nearby clusters are calculated in a range of radii from $100 \mathrm{kpc}$ to $500 \mathrm{kpc}$ only. Solid lines with $\bullet$ symbol are plotted for distant clusters, and dashed lines with $\times$ symbol are plotted for nearby clusters.

the morphology parameters in the radius sequence of $100 \mathrm{kpc}$ to $1 \mathrm{Mpc}$ for distant clusters $(z>0.05)$, while $100 \mathrm{kpc}$ to $500 \mathrm{kpc}$ for nearby clusters $(z<0.05)$. Figure 9 illustrates our result. As per this plot, in the case of distant clusters, a few parameters (asymmetry, smoothness, $G_{M}$, and ellipticity) remain constant in spite of aperture size, while the Gini, $M_{20}$, and concentration parameters are sensitive to the aperture radius within which they are calculated, while tending to be stable for aperture radii greater than $400 \mathrm{kpc}$. The Gini parameter increases with radius as more (faint) sky pixels are included in the extraction aperture. Parameter values for nearby clusters (in particular $z<0.05$ ) are very sensitive to the chosen aperture size.

\subsection{Exposure time effect}

It is important to check the consistency of the parameters over different exposure times. Observations with a shorter exposure time are likely to have lower $\mathrm{S} / \mathrm{N}$. Consequently, we were able to check the robustness of the parameters for cluster images with lower S/N. However, to rescale the real data by exposure time and then add Poisson noise gives an image that has an excessive amount of Poisson noise, in addition to the intrinsic noise present in the real observation. The simplest solution is to simulate a cluster image with no intrinsic noise, rather than using real data. To achieve this, we needed to estimate the different complex characteristics of a model to simulate galaxy clusters.
This task is considered difficult in the cases of non-relaxed and disturbed clusters. To overcome this problem, Hashimoto et al. (2007) suggest a novel technique for galaxy cluster simulations called "adaptive scalings", using real data and adding noise to the rescaled image. We refer the reader to Hashimoto et al. (2007), for more details about this technique.

An example of this adaptive method is given in Fig. 10 with an example of the low exposure time effect on a cluster. Subsequently we calculated our morphology parameters for each short-to-long-exposure cluster image.

Figure 11 shows how morphology parameters behave with different exposure lengths. We simulated a few original clusters with various exposure times, as described above, and re-calculated the morphology parameters for each simulated cluster. As depicted in Fig. 11, all parameters are robust against different exposure times, with the exception of the smoothness parameter. We noted, however, that the Gini, Gini of the secondorder moment, and asymmetry were not reliable when the exposure time was $\lesssim 5 \mathrm{ks}$. In the cases of the Gini and Gini of the second-order moment, low exposure means low $\mathrm{S} / \mathrm{N}$, indicating that the Gini and Gini of the second-order moment values are high for low-exposure observations, although fairly consistent for exposure times $\gtrsim 5 \mathrm{ks}$. For the short exposure times, there are a few bright pixels within the given aperture radius (the rest are scattered to very low $\sim 0$ ). In other words, low $\mathrm{S} / \mathrm{N}$ causes broader flux distribution in the faintest pixels, resulting in strong variation in the flux distribution, for which we 


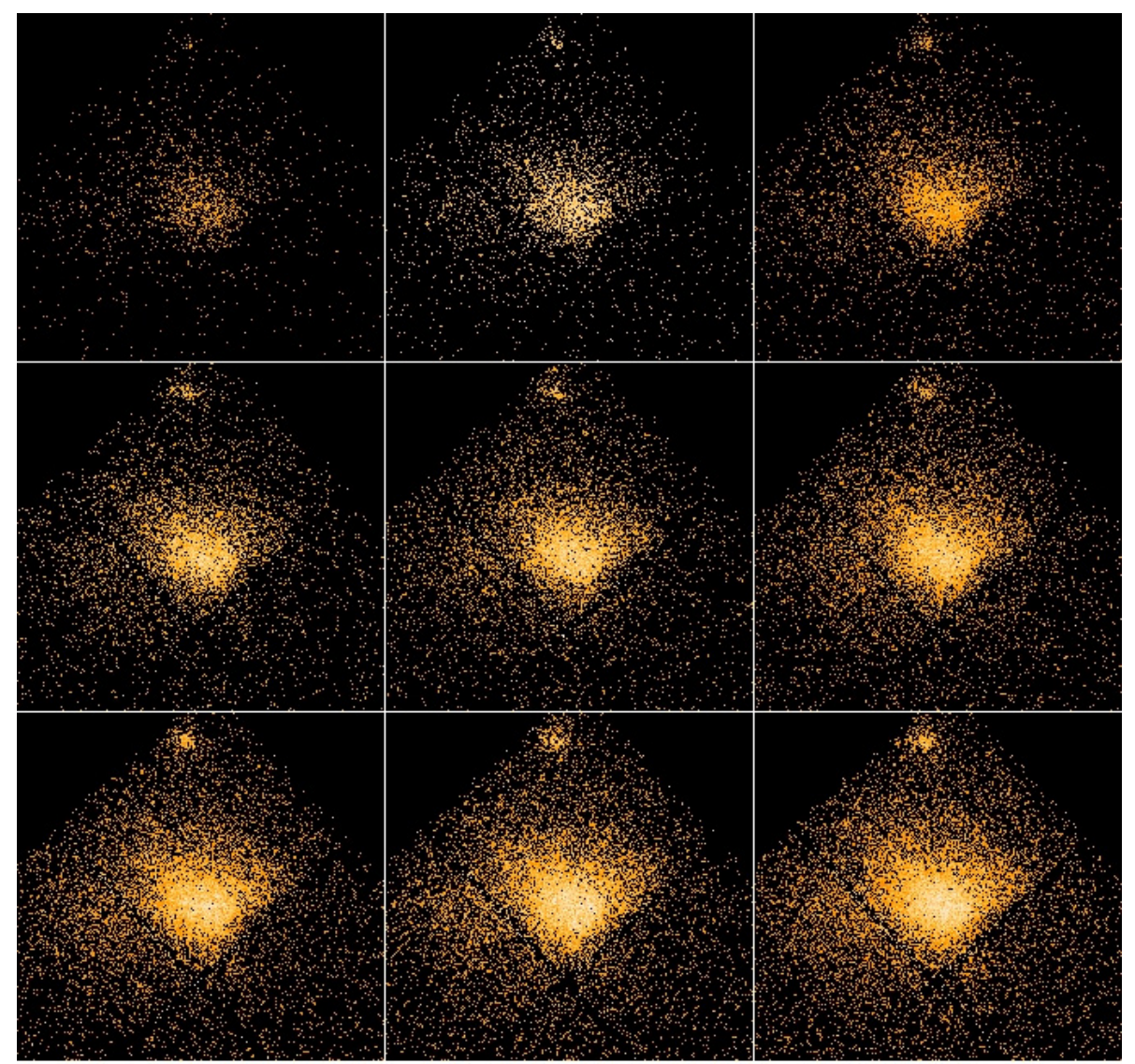

Fig. 10. Simulated clusters for different exposure times. Horizontally, from top left to bottom right, the images were arranged from $2 \mathrm{ks}$ to $18 \mathrm{ks}$, respectively, in order of $2 \mathrm{ks}$ exposure time.

calculated the Gini coefficient; as a result, the Gini coefficient gives a high value compared with the long exposure time. The value of the smoothness decreases continuously with increasing exposure time. This is to be expected because, for high $\mathrm{S} / \mathrm{N}$ and exposure time, the flux distribution in any given cluster image becomes smooth and less patchy. Care must therefore be taken when calculating the smoothness parameter. We found that the smoothness is weakly correlated with the Gini of the secondorder moment and asymmetry parameters, yet it is not established whether we can use the latter parameters as a substitute for the smoothness. The remainder of the parameters were relatively constant over different exposure lengths.

\subsection{Redshift effect}

It is hoped that these morphology parameters could be used to trace the evolution of galaxy clusters with redshifts. It is therefore important to understand the robustness as a function of redshift, and to this end, it is necessary to check the following criteria:

1. The effect of various angular bin sizes on morphology parameters.

2. The surface brightness dimming effect on morphology parameters.

We simulated several observations of galaxy clusters at a higher redshift $\left(z_{1}\right)$ than its real redshift $\left(z_{0}\right)$ using real data from our sample clusters. Again, we adopted the procedure describe by Hashimoto et al. (2007).
In Fig. 12 we give an example of clusters simulated via the method described above that illustrates how a cluster appears at high redshift. Subsequent to the simulation, we calculated morphology parameters for each cluster redshift and plotted them (Fig. 13).

We simulated a few original clusters with various redshifts, using the method described above, and re-calculated morphology parameters for each simulated cluster. Figure 13 illustrates that the smoothness is systematically increased with redshift. The reason could be that high- $z$ clusters are noisy and appear patchy. The ellipticity parameter appears particularly noisy. Except for a few clusters and after $z \gtrsim 0.5$, the Gini, $M_{20}$, concentration, asymmetry and Gini of the second- order moment parameters are fairly constant, and the systematics caused by redshifts are small.

\section{Comparison of parameters with X-ray gas properties}

We expect that the dynamical states of clusters to be influence by factors like merger histories, which in turn could influence a number of properties, such as luminosity, mass, temperature structure, and cooling flows. To test the potential of our parameters as probes of the physical conditions in clusters, we investigated the parameter correlations with source redshift, global $\mathrm{X}$-ray properties, cooling times and the presence of diffuse radio continuum emission. 

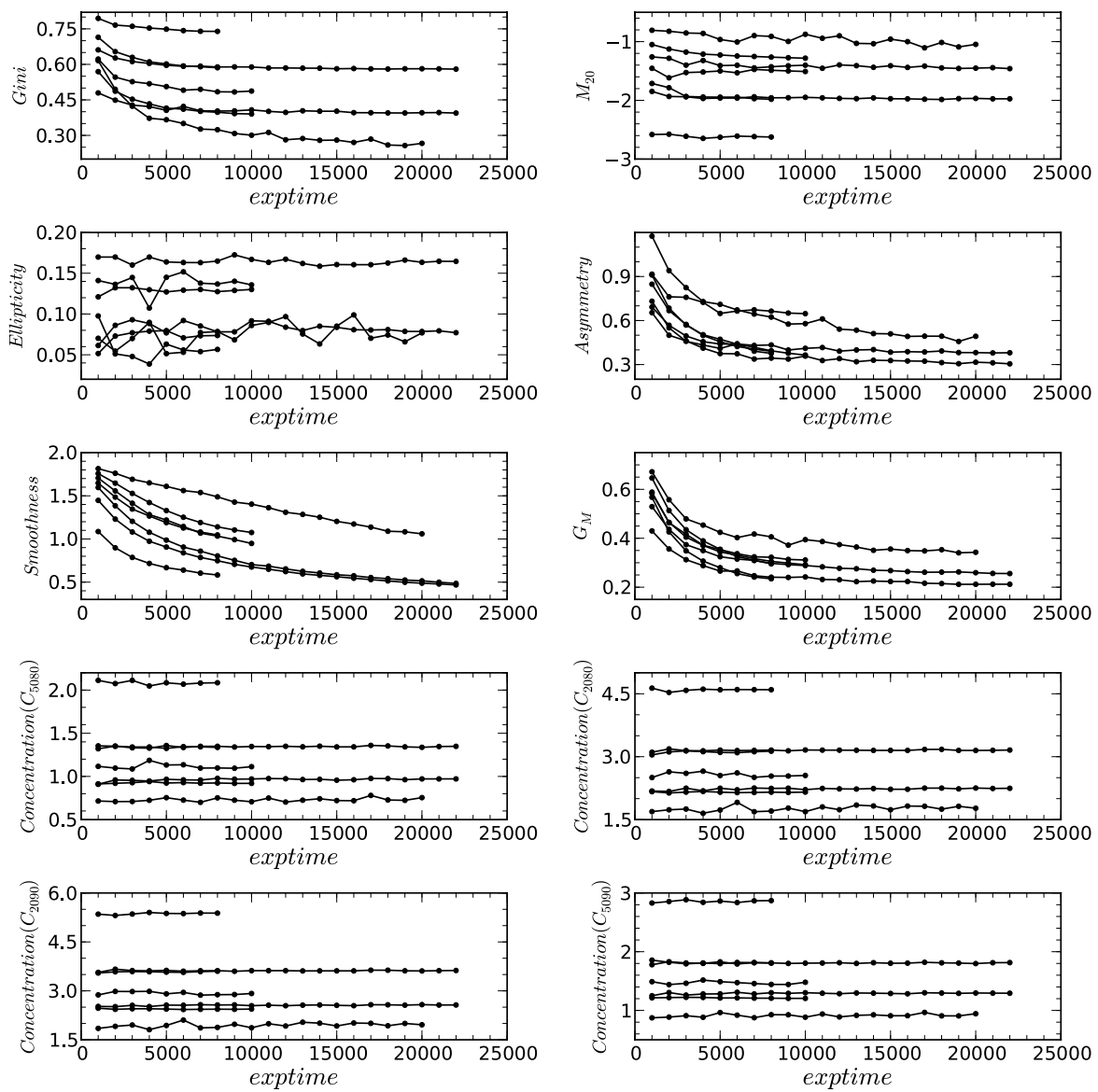

Fig. 11. Robustness of morphology parameters calculated for several clusters against various simulated exposure lengths.

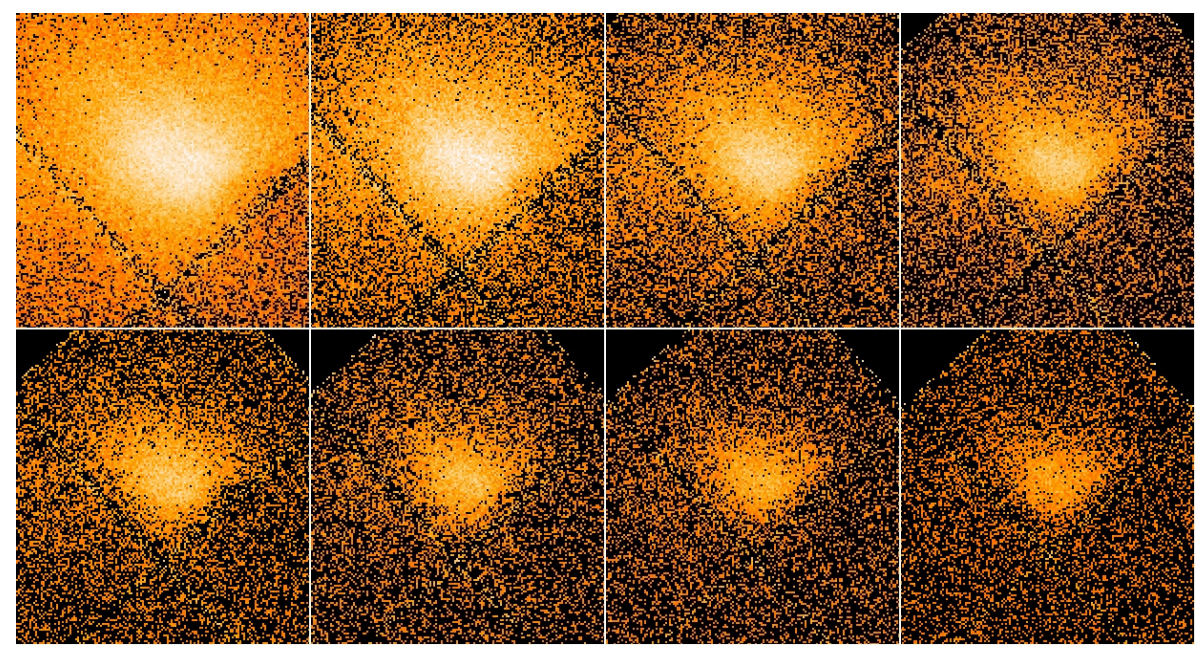

Fig. 12. Simulated clusters for different redshifts. Figures are read from top left to bottom right in order of low- to high-z, respectively.

\subsection{Redshift evolution}

According to the concordance model, massive galaxy clusters start to form around $z \sim 1$ and continue to evolve up to the present epoch. To look into the evolutionary effect on the distribution of the parameters, we divided our entire sample into low- $z$ $(0.02-0.3)$ and high- $z(0.3-0.9)$ clusters and classified the samples based on our morphology parameters. We also performed the K-S and R-S tests on each parameter distribution to observe the difference between the two redshift bins. Table 5 lists the mean, median, and statistical test results for the two redshift bins.

Below are more descriptions for the results of our three most promising parameters (viz. Gini, $M_{20}$, and concentration). The combination of the three parameters are plotted in Fig. 14, with boundaries between relaxed and non-relaxed clusters.

1. Based on the Gini, we found that 9 clusters are relaxed, 48 are intermediate and 27 are non-relaxed. In the low$z$ subsample there are $8(\sim 17 \%)$ relaxed, 25 intermediate $(\sim 52 \%)$, and $15(\sim 31 \%)$ non-relaxed clusters. In the high- $z$ 
V. Parekh et al.: Morphology parameters: substructure identification in X-ray galaxy clusters
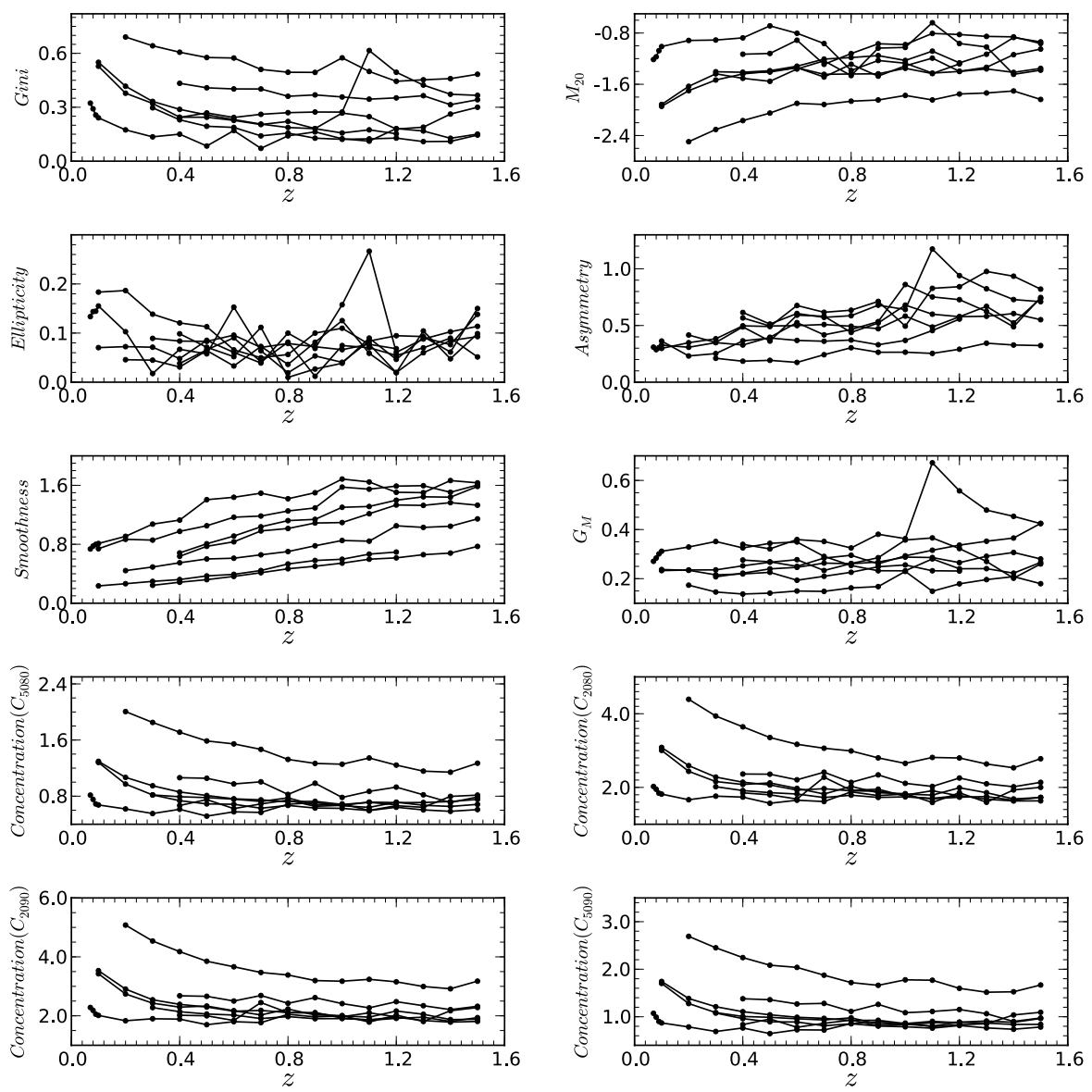

Fig. 13. Robustness of morphology parameters that are calculated for several clusters against simulated clusters at different redshifts.

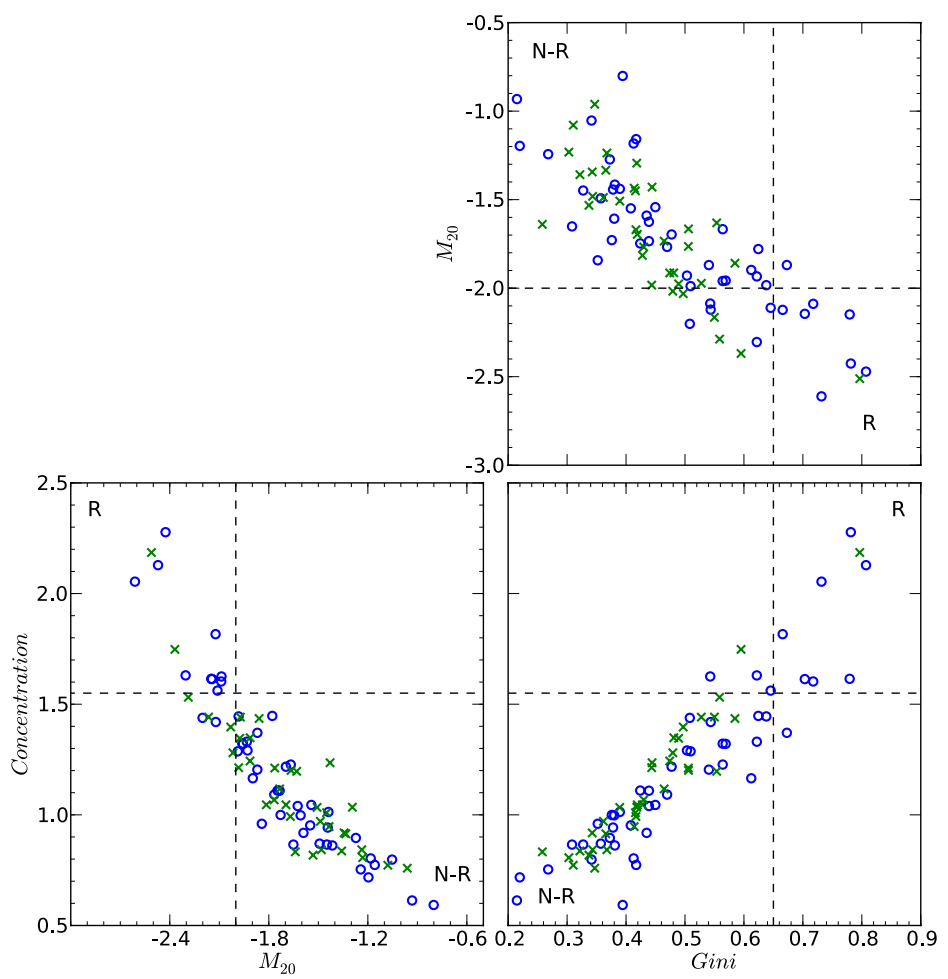

Fig. 14. Three parameters plotted in the parameter-parameter planes to show cluster evolution with redshift. Here we plotted $C_{5080}$ as the concentration parameter. $\circ=$ low- $z$ cluster $(0.02-0.3) ; \times=$ high- $z$ cluster $(0.3-0.9)$. The dashed lines represent the boundaries between relaxed and non-relaxed clusters. Boundary values for Gini $=0.65, M_{20}=-2.0$, and concentration $=1.55$. $\mathrm{R}$ indicates relaxed clusters and $\mathrm{N}-\mathrm{R}$ indicates non-relaxed clusters. 
Table 5. Statistics for two redshift bins: low- $z(0.02-0.3)$ and high- $z(0.3-0.9)$.

\begin{tabular}{ccccccc}
\hline \hline Parameters & Mean of low- $z$ & Mean of high- $z$ & Median of low- $z$ & Median of high- $z$ & K-S probability & R-S probability \\
\hline Gini & 0.50 & 0.44 & 0.47 & 0.43 & 0.08 & 0.09 \\
$M_{20}$ & -1.75 & -1.68 & -1.76 & -1.67 & 0.74 & 0.35 \\
Concentration & 1.21 & 1.14 & 1.14 & 1.06 & 0.57 & 0.47 \\
Asymmetry & 0.38 & 0.46 & 0.36 & 0.46 & 0.0002 & 0.0008 \\
Smoothness & 0.65 & 1.11 & 0.53 & 1.16 & $1.57 \times 10^{-7}$ & $2 \times 10^{-6}$ \\
$G_{M}$ & 0.28 & 0.31 & 0.28 & 0.30 & 0.0016 & 0.004 \\
Ellipticity & 0.10 & 0.08 & 0.09 & 0.07 & 0.08 & 0.024 \\
\hline
\end{tabular}

subsample, there are $1(\sim 3 \%)$ relaxed, $23(\sim 64 \%)$ intermediate, and $12(\sim 33 \%)$ non-relaxed clusters.

2. Based on the $M_{20}$, we found that 18 clusters are relaxed, 50 are intermediate and 16 are non-relaxed. In the low$z$ subsample there are $12(\sim 25 \%)$ relaxed, 28 intermediate $(\sim 58 \%)$, and $8(\sim 17 \%)$ non-relaxed clusters. In the high- $z$ subsample, there are $6(\sim 17 \%)$ relaxed, $22(\sim 61 \%)$ intermediate, and $8(\sim 22 \%)$ non-relaxed clusters.

3 . Based on the concentration, we found 12 clusters are relaxed, 41 clusters are intermediate, and 31 clusters are non-relaxed. In the low $-z$ subsample there are $10(\sim 21 \%)$ relaxed, 20 intermediate $(\sim 42 \%)$, and $18(\sim 37 \%)$ non-relaxed clusters. In the high- $z$ subsample, there are $2(\sim 6 \%)$ relaxed, $21(\sim 58 \%)$ intermediate, and $13(\sim 36 \%)$ non-relaxed clusters.

As seen in Table 5, the K-S and R-S probabilities are $<1 \%$ and $<0.1 \%$, respectively, for the asymmetry and smoothness parameters, implying that we can reject both null hypotheses mentioned in Sect. 4.1. Clearly most of the clusters in our sample are in the intermediate stage ( $\sim 57 \%$ based on the Gini coefficient, $\sim 60 \%$ based on the $M_{20}$, and $\sim 49 \%$ based on the concentration parameter). Weak evolution is visible in the Gini and Gini of the secondorder moment, which indicates the possibility that high redshift clusters are more extended (which could mean that they do not have a density peak at the cluster centre) compared with those of low redshift. The $M_{20}$, concentration, and ellipticity do not show any significant evolution. From our results, there is indication that relaxed clusters are more dominant within the low- $z$ sample, which could indicate that, in the current epoch, clusters show less substructure and are (fully) evolved as compared to distant clusters, but these results are marginal.

\subsection{X-ray luminosity, temperature and mass}

We compared seven morphology parameters with three global cluster properties (luminosity, temperature, and mass) taken from V09 to search for any possible correlation of these global properties with cluster morphology. Figure 15 shows a comparison, while Table 6 lists the Spearman coefficient values calculated for the clusters' global properties and galaxy cluster morphologies. Figure 15 defines each clusters' dynamical state according to the combination of the Gini, $M_{20}$, and concentration morphology parameters (Sect. 4.3).

No obvious correlation between cluster morphology and $\mathrm{X}$-ray global properties was found during our analysis. This may be because quantities such as X-ray luminosity and temperature are not solely related to the dynamical state of clusters (Hashimoto et al. 2007). These properties also depend strongly on cluster mass and on non-gravitational processes, such as supernovae feedback and central AGN heating (Donnelly et al. 1999; Tozzi \& Norman 2001; Neumann et al. 2003). Buote \& Tsai (1996) also compared their power ratio measurements for
Table 6. Spearman coefficient, $\rho$, for morphology parameters and X-ray global properties (luminosity, temperature, and mass).

\begin{tabular}{cccc}
\hline \hline & Luminosity & Temperature & Mass \\
\hline Gini & 0.19 & 0.03 & 0.03 \\
$M_{20}$ & -0.13 & 0.03 & 0.07 \\
Concentration & 0.18 & -0.03 & -0.05 \\
Asymmetry & -0.22 & -0.23 & -0.25 \\
Smoothness & -0.33 & -0.26 & -0.30 \\
$G_{M}$ & -0.25 & -0.17 & -0.21 \\
Ellipticity & 0.26 & 0.30 & 0.35 \\
\hline
\end{tabular}

X-ray clusters with the ICM temperature and luminosity, without finding any strong correlation between sub-clustering and global $\mathrm{X}$-ray properties. In agreement with our conclusion, they pointed out that this lack of correlation is reasonable, since power ratios are a measure of cluster evolution that do not take the cluster mass into account, to which all the other X-ray quantities (e.g. luminosity and temperature) are sensitive. We observed that all relaxed clusters occupy high Gini, low $M_{20}$, and high concentration values in all three plots. We therefore concluded that, to identify the dynamical state of a galaxy cluster, X-ray global properties are not as useful as the surface brightness distribution or cluster morphology, which is quantifiable using morphology parameters.

\subsection{X-ray cluster cooling time}

$\mathrm{X}$-ray emission is considered to be the primary cooling process for the ICM. The cooling time is much shorter than the Hubble time at the centre of a cluster, allowing gas to cool from a high ICM temperature $\sim 10^{8} \mathrm{~K}$ down to $\sim 10^{7} \mathrm{~K}$. Numerical simulations suggest that cluster mergers may disturb gas cooling (Ritchie \& Thomas 2002; Burns et al. 2008; Hudson et al. 2010). We aimed to investigate the relationship between the degree of substructure and cooling times or rates of a cluster. We used the cooling time information supplied by Hudson et al. (2010). Table 7 lists the cooling time values for low- $z$ clusters.

Of the relaxed systems (as identified by the V09), A3158, A3391, ZwCl1215, A3562, and A401 have relatively high cooling time values. These systems were classified as nonrelaxed clusters by our combination of morphology parameters. In the non-relaxed sample (again from the V09), only three clusters - A3558, A1644, and A2065 - have relatively short cooling times. The short cooling times may suggest that the cores of these clusters might not yet be disturbed by cluster merger. Rossetti et al. (2007) analysed the Chandra and XMM observations of A3558 and find that its cool core had survived a merger. The Chandra observations show bright cluster nuclei, which also supports this idea. Chatzikos et al. (2006) show that A2065 is an unequal mass merger, which could be a reason 
V. Parekh et al.: Morphology parameters: substructure identification in X-ray galaxy clusters
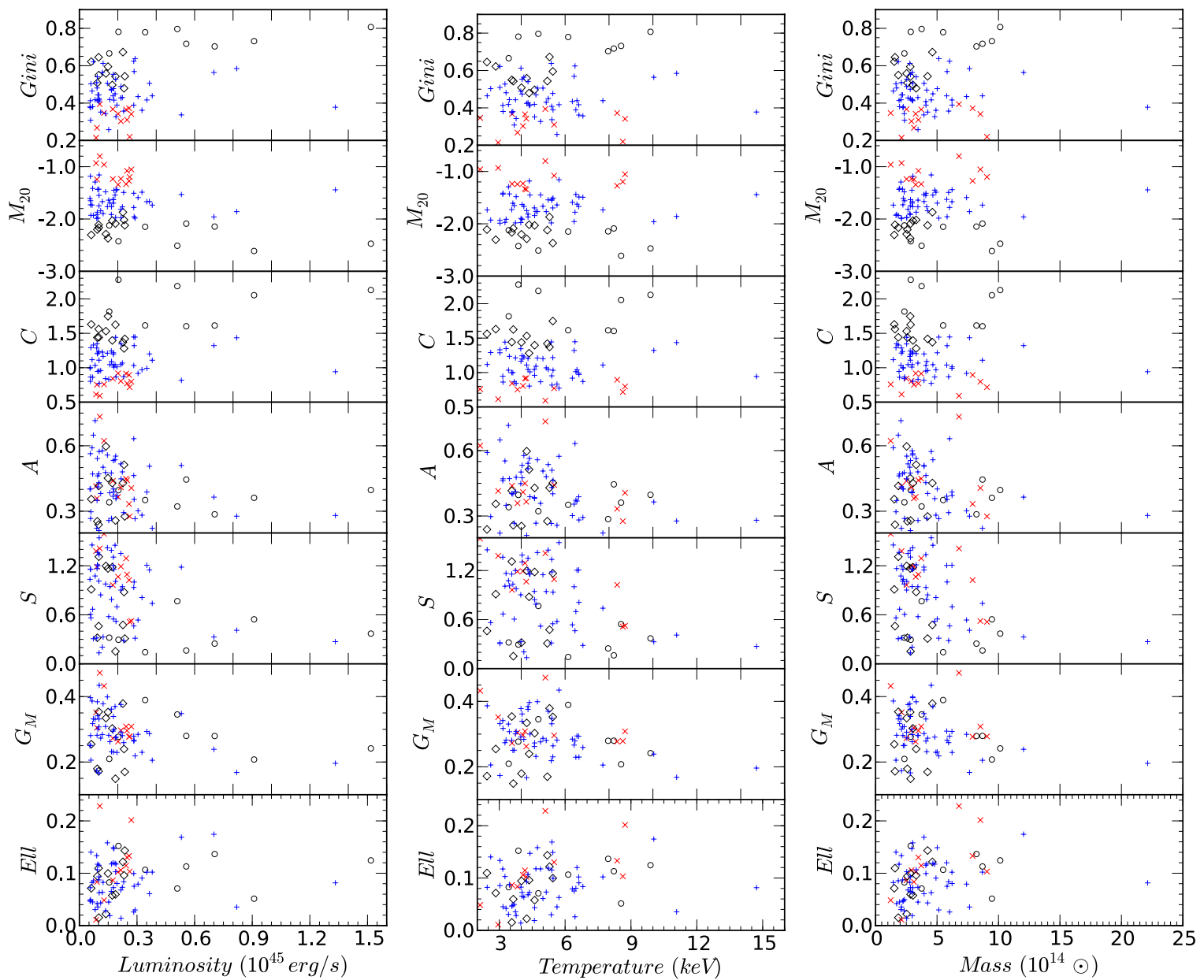

Fig. 15. Comparison of luminosity (left), temperature (middle), and mass (right) (estimated from the $Y_{X}$ parameter) with morphology parameters. $\circ=$ strong relaxed clusters; $\diamond=$ relaxed clusters; $+=$ non-relaxed clusters; and $\times=$ strong non-relaxed clusters. We plotted $C_{5080}$ as the concentration parameter. We take all these global properties (luminosity, temperature, and mass) values from the V09.

behind the survival of one of its cool cores. According to the classical definition of a cooling flow, $t_{\text {cool }}<t_{\text {age }}$, where $t_{\text {age }}$ (age of galaxy cluster) $\sim 10 \mathrm{Gyr}$. We found the mean $t_{\text {cool }}$ value for strong relaxed clusters to be $0.44 \mathrm{Gyr}, 0.64 \mathrm{Gyr}$ for relaxed clusters, 4.72 Gyr for non-relaxed clusters, and 12.5 Gyr for strong non-relaxed clusters. This implies that the cooling mechanism is completely disturbed in strong non-relaxed clusters, while only $\sim 17 \%$ are completely disturbed among non-relaxed clusters (five non-relaxed clusters have $\left.t_{\text {cool }}>t_{\text {age }}\right)$. Unfortunately, we did not have cooling time information for the high- $z$ clusters in our sample.

The correlation of morphology parameters with cooling time is plotted in Fig. 16 for the concentration, Gini, and $M_{20}$ on a log-log scale. Figures 16a, 16b, and 16c show that two of our parameters, the concentration and Gini, are anti-correlated, while the $M_{20}$ is correlated with the cooling time of clusters. This indicates the possibility that surface brightness imaging data could be useful in deriving the cooling time information of the central intracluster gas using simple morphology parameters. To investigate linear fitting, we simply used the power law model to establish a relationship between the morphology parameters and $t_{\text {cool }}$. For all three parameters, we constituted $t_{\text {cool }} \propto$ Conc $^{-3.42 \pm 0.55}, t_{\text {cool }} \propto \mathrm{Gini}^{-3.0 \pm 0.35}$, and $t_{\text {cool }} \propto M_{20}^{4.23 \pm 0.32}$. Table 8 lists the Spearman coefficient, $\rho$, between the concentration, Gini, and $M_{20}$ parameters and cluster cooling time.

\subsection{Radio halo cluster dynamical states}

Previous studies have shown the importance of joint X-ray and radio data to study the origin of non-thermal radio emission from galaxy clusters, in the form of a cluster wide radio halo which is generally situated at the cluster centre (Feretti et al. 2012, and references therein). Current results indicate the presence of diffuse intracluster radio sources only in dynamically disturbed clusters, and it is expected that future radio surveys will reveal diffuse radio emission from a large number of major and minor mergers (Cassano et al. 2010a).

The relations between cluster properties derived from X-ray observations (luminosity $\left(L_{x}\right)$, temperature $(\mathrm{T})$, and mass $(\mathrm{M})$ ) and radio halo luminosity have been widely studied in the past few years (Cassano et al. 2007, 2011; Giovannini et al. 2009; Brunetti et al. 2009; Venturi 2011). They all show a strong correlation between radio halo and X-ray emission in galaxy clusters. Buote (2001), Schuecker et al. (2001), and Cassano et al. (2010b) show a relation between non-thermal radio sources and X-ray cluster morphology. Buote (2001) notes the linear relation between radio power $\left(P_{1.4 \mathrm{GHz}}\right)$ and power ratio $\left(P_{1} / P_{0}\right)$ (Buote \& Tsai 1995a, 1996) for ROSAT observed X-ray clusters. He concludes that approximately $P_{1.4 \mathrm{GHz}} \propto P_{1} / P_{0}$, which means the clusters that host the powerful radio haloes are experiencing the largest departures from a virialized state. Recently, 

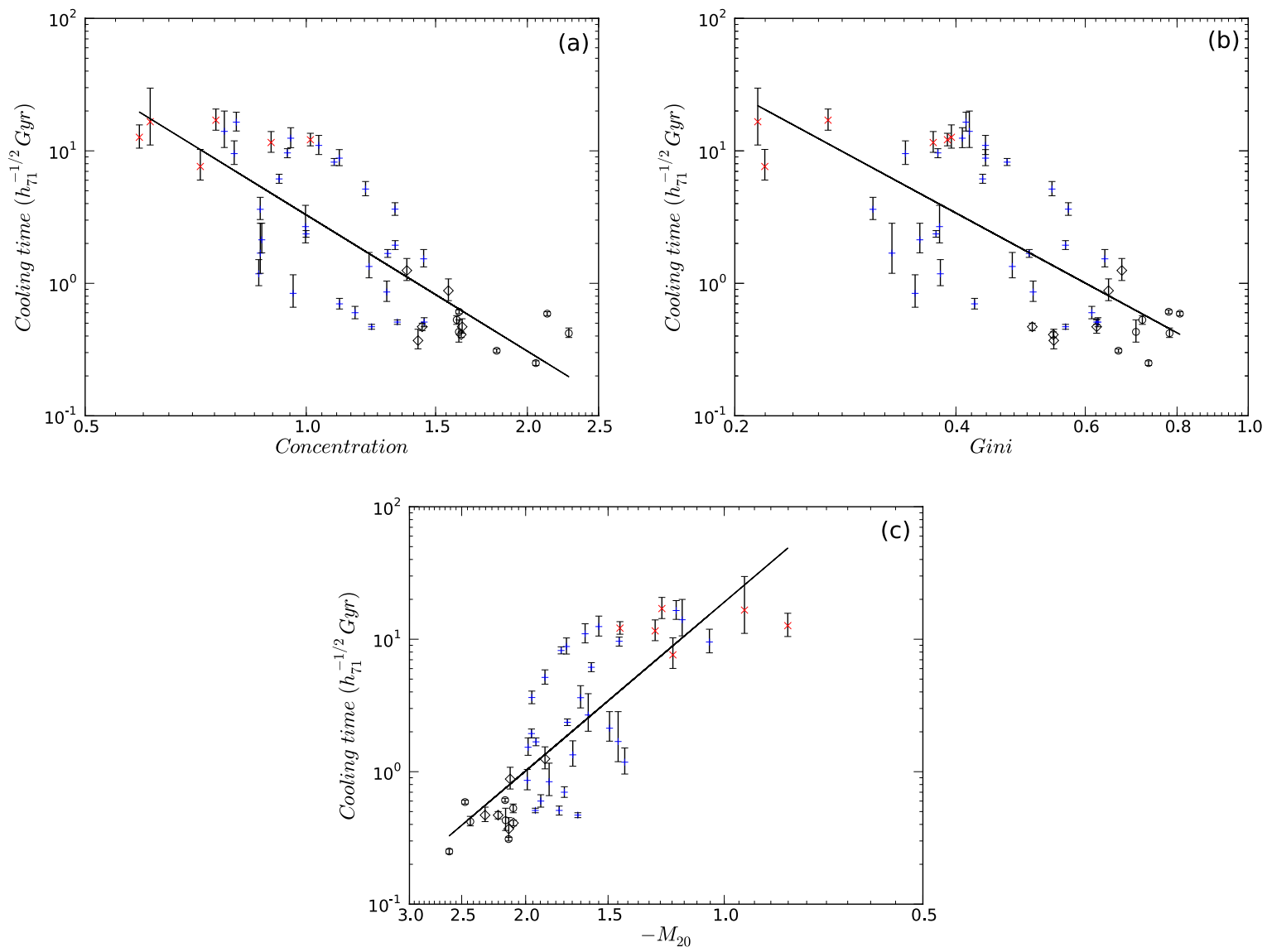

Fig. 16. Panel a): concentration $\left(C_{5080}\right)$ parameter negatively correlated with cooling time for fixed radii. Panel b): Gini coefficient negatively correlated with cooling time. Panel c): $M_{20}$ correlated with cooling time. $\circ=$ strong relaxed clusters; $\diamond=$ relaxed clusters; $+=$ non-relaxed clusters; and $\times=$ strong non-relaxed clusters. We used the power law fitting to show the linear correlation.

Table 7. Available cooling time, $t_{\text {cool }}$, values from (Hudson et al. 2010) for the low- $z$ clusters.

\begin{tabular}{|c|c|c|c|}
\hline Cluster name & $t_{\text {cool }}(\mathrm{Gyr})$ & Cluster name & $\overline{t_{\text {cool }}(\mathrm{Gyr})}$ \\
\hline A3571 & $2.13_{0.71}^{-0.43}$ & A2597 & $0.42_{0.04}^{-0.03}$ \\
\hline A2199 & $0.6_{0.07}^{-0.06}$ & A133 & $0.47_{0.03}^{-0.03}$ \\
\hline 2A 0335 & $0.31_{0.01}^{-0.01}$ & A2244 & $1.53_{0.27}^{-0.2}$ \\
\hline A496 & $0.47_{0.02}^{-0.02}$ & RXJ1504 & $0.59_{0.02}^{-0.02}$ \\
\hline A85 & $0.51_{0.04}^{-0.04}$ & A2204 & $0.25_{0.01}^{-0.01}$ \\
\hline A478 & $0.43_{0.1}^{-0.07}$ & A2029 & $0.53_{0.04}^{-0.04}$ \\
\hline A1795 & $0.61_{0.02}^{-0.02}$ & A 2142 & $1.94_{0.16}^{-0.14}$ \\
\hline A4038 & $1.68_{0.12}^{-0.11}$ & A 3562 & $5.15_{0.72}^{-0.57}$ \\
\hline A2052 & $0.51_{0.02}^{-0.02}$ & A401 & $8.81_{1.41}^{-1.08}$ \\
\hline Hydra-A & $0.41_{0.02}^{-0.02}$ & A 3558 & $1.69_{1.15}^{-0.5}$ \\
\hline A2063 & $2.36_{0.14}^{-0.13}$ & A2147 & $17.04_{3.64}^{-2.72}$ \\
\hline A 3158 & $8.22_{0.54}^{-0.47}$ & A 3266 & $7.62_{2.63}^{-1.64}$ \\
\hline MKW3s & $0.86_{0.18}^{-0.13}$ & A119 & $14.03_{5.95}^{-3.43}$ \\
\hline EXO0422 & $0.47_{0.07}^{-0.05}$ & A1644 & $0.84_{0.32}^{-0.18}$ \\
\hline A4059 & $0.7_{0.07}^{-0.06}$ & A1736 & $16.59_{13.17}^{-5.52}$ \\
\hline A2589 & $1.18_{0.33}^{-0.22}$ & A3395 & $12.66_{3.04}^{-2.18}$ \\
\hline A 3112 & $0.37_{0.08}^{-0.05}$ & A2065 & $1.34_{0.37}^{-0.04}$ \\
\hline A1651 & $3.63_{0.43}^{-0.37}$ & A 3667 & $6.14_{0.52}^{-0.45}$ \\
\hline A576 & $3.62_{0.84}^{-0.59}$ & A754 & $9.53_{2.37}^{-1.64}$ \\
\hline A2657 & $2.68_{1.2}^{-0.66}$ & A 2256 & $11.56_{2.43}^{-1.81}$ \\
\hline A3391 & $12.46_{2.49}^{-1.89}$ & A399 & $12.13_{1.44}^{-1.22}$ \\
\hline A1650 & $1.25_{0.29}^{-0.2}$ & A2163 & $9.65_{0.73}^{-0.78}$ \\
\hline S 1101 & $0.88_{0.2}^{-0.14}$ & A3376 & $16.47_{3.1}^{-2.35}$ \\
\hline ZwCl1215 & $10.99_{2.09}^{-1.61}$ & & \\
\hline
\end{tabular}

Table 8. Spearman coefficient, $\rho$, between morphology parameters and cluster cooling time.

\begin{tabular}{cc}
\hline \hline Morphology parameters & Cooling time (Gyr) \\
\hline Concentration & -0.82 \\
Gini & -0.71 \\
$M_{20}$ & 0.83 \\
\hline
\end{tabular}

Cassano et al. (2010b) have used three parameters, namely centroid shift (Mohr et al. 1993; Poole et al. 2006; Maughan et al. 2008; Böhringer et al. 2010), third-order power ratio $\left(P_{3} / P_{0}\right)$, and concentration (Santos et al. 2008) to demonstrate a relation between cluster mergers and the presence of a radio halo.

Since we have shown that our morphology parameters are useful for characterising the dynamical state of galaxy clusters, we investigate any possible correlation between our set of parameters and the presence of diffuse intracluster radio emission. We took 25 halo clusters from Giovannini et al. (2009) (as listed in Table 9) where some of them are already present in the V09 cluster sample (A754, A2256, A401, A3562, A399, and A2163). We reduced the X-ray data of the sample of Giovannini et al. (2009) in a similar way to what is described in Sect. 3.2. We subsequently calculated the morphology parameters (Gini, $M_{20}$, and concentration) for each cluster (see Table A.3).

In Fig. 17 we show that some parameters are useful for studying the dynamical state of radio halo clusters. In the bottom left- and right-hand plots, we can see that the radio halo clusters are separated from the relaxed clusters and overlap with 
Table 9. Radio halo sample clusters.

\begin{tabular}{|c|c|c|c|c|c|c|c|c|c|c|}
\hline Name & $z$ & kpc/" & $\begin{array}{c}S(1.4) \\
\text { mJy }\end{array}$ & $\begin{array}{l}\Delta S \\
\mathrm{mJy}\end{array}$ & $\begin{array}{c}\log P(1.4) \\
\mathrm{W} / \mathrm{Hz}\end{array}$ & $\begin{array}{l}\text { LLS } \\
\mathrm{Mpc}\end{array}$ & $\begin{array}{l}\text { Luminosity } \\
\left(10^{44} \mathrm{erg} / \mathrm{s}\right)\end{array}$ & $\begin{array}{c}\text { Temperature } \\
(\mathrm{keV})\end{array}$ & $\begin{array}{c}\text { Exposure time } \\
\text { ks }\end{array}$ & Ref. \\
\hline A1914 & 0.1712 & 2.80 & 64.0 & 3.0 & 24.04 & 1.01 & 9.86 & 10.50 & 19 & 1,2 \\
\hline A2218 & 0.1756 & 2.86 & 4.7 & 0.1 & 22.96 & 0.37 & 5.46 & 6.70 & 59 & 1,2 \\
\hline A665 & 0.1819 & 2.95 & 43.1 & 2.2 & 23.92 & 1.77 & 9.13 & 8.30 & 39 & 1,2 \\
\hline A520 & 0.1990 & 3.16 & 34.4 & 1.5 & 23.91 & 1.08 & 7.85 & 7.40 & 9 & 1,2 \\
\hline A773 & 0.2170 & 3.38 & 12.7 & 1.3 & 23.57 & 1.21 & 7.52 & 8.53 & 20 & 1,2 \\
\hline IE0657-56 & 0.2960 & 4.26 & 78.0 & 5.0 & 24.64 & 2.0 & 21.37 & 11.64 & 84 & 1,2 \\
\hline A2255 & 0.0806 & 1.46 & 56.0 & 3.0 & 23.28 & 0.8 & 2.50 & 6.42 & 39 & 1,2 \\
\hline A2319 & 0.0557 & 1.04 & 153.0 & 8.0 & 23.38 & 1.0 & 8.00 & 9.49 & 14 & 1,2 \\
\hline A754 & 0.0542 & 1.01 & 86.0 & 4.0 & 23.12 & 0.96 & 2.10 & 9.94 & 44 & 1,2 \\
\hline A2256 & 0.0581 & 1.08 & 103.4 & 1.1 & 23.26 & 0.79 & 3.55 & 6.90 & 12 & 1,2 \\
\hline A401 & 0.0737 & 1.34 & 17.0 & 1.0 & 22.70 & 0.50 & 6.17 & 8.07 & 18 & 1,2 \\
\hline A3562 & 0.0490 & 0.92 & 20.0 & 2.0 & 22.41 & 0.27 & 1.48 & 3.80 & 19 & 1,2 \\
\hline A399 & 0.0718 & 1.31 & 16.0 & 0 & 22.67 & 0.55 & 3.60 & 5.80 & 48 & 3,2 \\
\hline A2163 & 0.2030 & 3.22 & 155.0 & 2.0 & 24.57 & 2.21 & 21.50 & 12.12 & 71 & 1,2 \\
\hline A1300 & 0.3072 & 4.37 & 20.0 & 2.0 & 24.10 & 1.26 & 13.0 & 9.2 & 14 & 1,4 \\
\hline A1758 & 0.2790 & 4.08 & 16.7 & 0.8 & 23.93 & 1.47 & 6.70 & 7.95 & 7 & 1,2 \\
\hline A1995 & 0.3186 & 4.48 & 4.1 & 0.7 & 23.47 & 0.80 & 8.35 & 8.60 & 56 & 1,2 \\
\hline A2034 & 0.1130 & 1.97 & 13.6 & 1.0 & 23.00 & 0.59 & 3.60 & 7.15 & 195 & 1,2 \\
\hline A209 & 0.2060 & 3.25 & 16.9 & 1.0 & 23.65 & 1.36 & 5.84 & 8.28 & 20 & 1,2 \\
\hline A2219 & 0.2256 & 3.48 & 81.0 & 4.0 & 24.40 & 1.67 & 11.53 & 9.81 & 42 & 1,2 \\
\hline A2294 & 0.1780 & 2.90 & 5.8 & 0.5 & 23.06 & 0.52 & 3.70 & 7.10 & 10 & 1,2 \\
\hline A2744 & 0.3080 & 4.38 & 57.1 & 2.9 & 24.55 & 1.84 & 12.16 & 9.61 & 24 & 1,2 \\
\hline A521 & 0.2533 & 3.80 & 5.9 & 0.5 & 23.40 & 1.14 & 8.01 & 6.74 & 37 & 1,2 \\
\hline A697 & 0.2820 & 4.11 & 7.8 & 1.0 & 23.62 & 0.63 & 9.84 & 9.06 & 27 & 1,2 \\
\hline RXCJ2003.5-2323 & 0.3171 & 4.46 & 35.0 & 2.0 & 24.40 & 1.36 & 8.63 & 9.1 & 50 & 1,5 \\
\hline
\end{tabular}

Notes. Columns: (1) cluster name; (2) redshift; (3) conversion factor (angular size to linear size); (4) radio flux density (1.4 GHz); (5) error in estimated radio flux density; (6) total radio power (1.4 GHz); (7) radio largest linear size; (8) total X-ray luminosity (0.1-2.4 keV); (9) temperature (keV); (10) exposure time; (11) references. We normalised $H$ dependent quantities with $H_{0}=73 \mathrm{~km} \mathrm{~s}^{-1} \mathrm{Mpc}^{-1}$.

References. 1 = Giovannini et al. (2009, and references therein); 2 = Cavagnolo et al. (2009); 3 = Feretti et al. (2012, and references therein); $4=$ Ziparo et al. (2012); 5 = Giacintucci et al. (2009).

non-relaxed clusters in $M_{20}$ and Gini vs. concentration parameter planes, respectively (similar results were observed by Cassano et al. 2010b; Buote 2001). In these plots, we can roughly separate relaxed and non-relaxed clusters based on $M_{20}$ and concentration, where the region contained by $M_{20}<-2.0$ and concentration $>1.55$ gives exclusively relaxed clusters. Based on the Gini and concentration plot, all non-relaxed clusters have Gini $<0.65$ and concentration $<1.55$. The upper left- and right-hand plots in Fig. 17 show $M_{20}$ vs. temperature and Gini vs. temperature, respectively. We subdivided the $M_{20}$ vs. temperature plot into three regions: (1) the $M_{20}<-2.0$ region has all dynamically relaxed clusters; (2) the $M_{20}>-2.0$ and temperature $<6 \mathrm{keV}$ region has radio quiet merger clusters; and (3) the $M_{20}>-2.0$ and temperature $>6 \mathrm{keV}$ region has radio loud merger clusters. Similarly, the Gini vs. temperature plot has three regions defined as (1) the Gini $>0.65$ region has only dynamically relaxed clusters; (2) the Gini $<0.65$ and temperature $<6 \mathrm{keV}$ region has radio quiet merger clusters; and (3) the Gini $<0.65$ and temperature $>6 \mathrm{keV}$ region has radio loud merger clusters.

\section{Discussion and conclusion}

In this work we used a set of morphological parameters, among which some have so far only been applied to quantifying galaxy morphologies to obtain constraints on the dynamical state of clusters. These parameters do not depend on any model parameter fits, such as the beta model or power law, and can therefore be applied to both disturbed and regular clusters. In principle, the Gini, concentration, and $M_{20}$ are promising for the detection of disturbances in clusters at any scale. To measure the concentration, it was necessary to fix the cluster centre position (optical position of the BCG, X-ray peak, or barycentre) and then define the inner and outer regions around the chosen centre. This is not always possible in many of the high $-z(\sim 1)$ clusters due to low photon counts and distorted cluster morphology. In this work, we showed a tight correlation between the concentration and the Gini coefficient for both the low- and high- $z$ clusters. This suggested that the Gini coefficient could be used as a replacement for the concentration mainly for high- $z$ clusters. The chief benefit of using the Gini coefficient is that we do not need a well-defined cluster centre. It works well for low photon counts and also accurately quantifies substructure. This coefficient is completely independent of cluster shape, does not depend on any symmetry underlying the cluster, and is independent of the location of projected (bright) pixels (whether they be at the centre or the edge) in the given aperture.

The Gini coefficient was also correlated with the $M_{20}$, which could suggest that, in a relaxed system, $20 \%$ of the X-ray flux is centrally located where the electron density is high, while this is not possible for distorted and non-relaxed clusters where mergers may change the cluster density distribution. Most relaxed and cool-core clusters have Gini $>0.65$, which means that most of the flux comes from only a few bright pixels, mainly found at the cluster centre. Weak cool-core clusters were found between $0.4<$ Gini $<0.65$, and most disturbed clusters had Gini $<0.4$. This is obvious because, in non-relaxed clusters, the X-ray emission (or bright pixels) is not compact but equally distributed in the given aperture radius, which gives a low Gini value. 

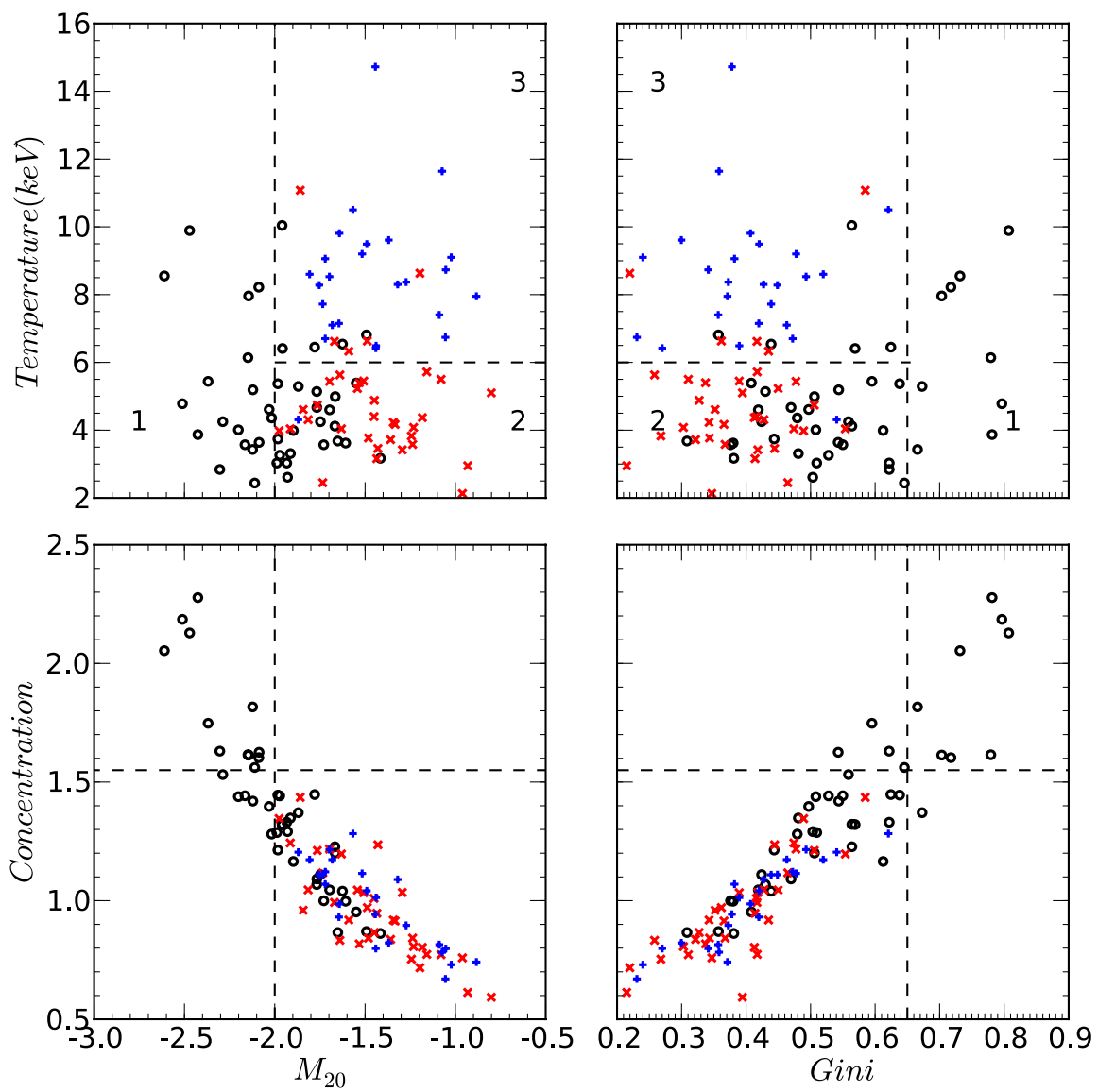

Fig. 17. Bottom left and right panels: $M_{20}$ and Gini vs. concentration, respectively. Top left and right panels: $M_{20}$ and Gini vs. temperature, respectively. $\circ=$ relaxed cluster; and $\times=$ non-relaxed cluster. Radio halo clusters are identified with a "+" symbol. Galaxy cluster separation is based on the V09.

We found that, unlike the Gini, $M_{20}$, and concentration parameters, the smoothness and asymmetry parameters did not show any reliable signs of being able to classify galaxy clusters based on their dynamical state. This was unexpected because these parameters trace the 2D structure and should be sensitive to a range of substructure types. In addition, Rasia et al. (2013) found that the smoothness \& asymmetry were promising as probes for substructure. Rasia et al. (2013), however, tested their parameters on simulated cluster data, and all the simulations had a uniform exposure time. We found that the value of these two parameters largely depends on cluster exposure time or $\mathrm{S} / \mathrm{N}$. We have also shown that, in clusters samples that have heterogeneous exposure times, as in our situation, the asymmetry and smoothness are not reliable parameters. Our sample does not have uniform exposure time for all clusters, which largely affects $\mathrm{S} / \mathrm{N}$ and photon counts between clusters.

We did not find any relationship between the morphology parameters and X-ray global properties of galaxy clusters. This is most likely because morphology parameters are estimated without regard for the mass of clusters, to which other quantities, such as X-ray luminosity and temperature, are instead related. The Gini, concentration, and $M_{20}$ appear to be correlated with the cooling time of clusters. X-ray imaging and surface brightness maps are therefore useful in investigating the cooling time of relaxed clusters.

The morphology parameters studied in this paper were robust in various physical or observational conditions (high redshift, low exposure, etc.). They helped us to investigate cluster morphology and dynamical state with sufficient accuracy. We noticed, however, that the Gini, Gini of the second-order moment, and asymmetry are fairly weak parameters at $<5 \mathrm{ks}$ exposure time. The smoothness parameter systematically decreases with increasing exposure time and is therefore not suitable when comparing objects with a range of redshifts and exposure times. The concentration and $M_{20}$ are reasonably robust at low exposure times. Systematics associated with redshift effects are low, but some caution is required for interpreting the results based on simulated clusters. We concluded that the concentration and $M_{20}$ are more robust than the other parameters. We also tested the Gini, $M_{20}$, and concentration parameters against different background level and found that these parameters are robust.

We have taken 25 radio loud clusters, which are well-known non-relaxed clusters that host radio haloes. Our morphology parameters are quite useful for studying their dynamical activities. Based on Gini, $M_{20}$, and concentration parameters and in agreement with previous results (Cassano et al. 2010b), we find that only - but not all - merging systems host radio haloes. We can approximately separate radio halo clusters from relaxed clusters in parameter space with concentration $\left\langle 1.5, M_{20}>-2.0\right.$, and Gini $<0.65$.

In conclusion, we have shown that the combination of Gini, concentration, and $M_{20}$ show a strong potential for identifying substructure and perturbed dynamical states. In the future we will also investigate scaling relations between cluster mass and luminosity or temperature by dividing clusters based on parameter boundary values. 
Acknowledgements. V. Parekh acknowledges financial support from the South African Square Kilometer Array Project. C. Ferrari acknowledges financial support by the "Agence Nationale de la Recherche" through grant ANR-09-JCJC0001-01. We are grateful to Prof. Alexei Vikhlinin (CFA) for providing the Chandra archival data. G. Angus acknowledges support from the Claude-Leon foundation.

\section{References}

Abraham, R. G., van den Bergh, S., \& Nair, P. 2003, ApJ, 588, 218

Andrade-Santos, F., Lima Neto, G. B., \& Laganá, T. F. 2012, ApJ, 746, 139

Bershady, M. A., Jangren, A., \& Conselice, C. J. 2000, AJ, 119, 2645

Böhringer, H., Pratt, G. W., Arnaud, M., et al. 2010, A\&A, 514, A32

Brunetti, G., Cassano, R., Dolag, K., \& Setti, G. 2009, A\&A, 507, 661

Buote, D. A. 2001, ApJ, 553, L15

Buote, D. A., \& Tsai, J. C. 1995a, ApJ, 452, 522

Buote, D. A., \& Tsai, J. C. 1995b, ApJ, 439, 29

Buote, D. A., \& Tsai, J. C. 1996, ApJ, 458, 27

Burenin, R. A., Vikhlinin, A., Hornstrup, A., et al. 2007, ApJS, 172, 561

Burns, J. O., Hallman, E. J., Gantner, B., Motl, P. M., \& Norman, M. L. 2008, ApJ, 675, 1125

Cassano, R., Brunetti, G., Setti, G., Govoni, F., \& Dolag, K. 2007, MNRAS, 378, 1565

Cassano, R., Brunetti, G., Röttgering, H. J. A., \& Brüggen, M. 2010a, A\&A, 509, A68

Cassano, R., Ettori, S., Giacintucci, S., et al. 2010b, ApJ, 721, L82

Cassano, R., Brunetti, G., \& Venturi, T. 2011, JA\&A, 32, 519

Cavagnolo, K. W., Donahue, M., Voit, G. M., \& Sun, M. 2009, ApJS, 182, 12

Chatzikos, M., Sarazin, C. L., \& Kempner, J. C. 2006, ApJ, 643, 751

Conselice, C. J. 2003, ApJS, 147, 1

Donnelly, R. H., Markevitch, M., Forman, W., et al. 1999, ApJ, 513, 690

Dupke, R. A., Mirabal, N., Bregman, J. N., \& Evrard, A. E. 2007, ApJ, 668, 781

Feretti, L., Giovannini, G., Govoni, F., \& Murgia, M. 2012, A\&ARv, 20, 54

Ferrari, C., Arnaud, M., Ettori, S., Maurogordato, S., \& Rho, J. 2006, A\&A, 446, 417

Ferrari, C., Govoni, F., Schindler, S., Bykov, A. M., \& Rephaeli, Y. 2008, Space Sci. Rev., 134, 93

Giacintucci, S., Venturi, T., Brunetti, G., et al. 2009, A\&A, 505, 45

Giovannini, G., Bonafede, A., Feretti, L., et al. 2009, A\&A, 507, 1257

Hashimoto, Y., Böhringer, H., Henry, J. P., Hasinger, G., \& Szokoly, G. 2007, A\&A, 467, 485

Holwerda, B. W., Pirzkal, N., Cox, T. J., et al. 2011a, MNRAS, 416, 2426

Holwerda, B. W., Pirzkal, N., de Blok, W. J. G., et al. 2011b, MNRAS, 416, 2437
Holwerda, B. W., Pirzkal, N., de Blok, W. J. G., et al. 2011c, MNRAS, 416, 2401

Hudson, D. S., Mittal, R., Reiprich, T. H., et al. 2010, A\&A, 513, A37

Jeltema, T. E., Canizares, C. R., Bautz, M. W., \& Buote, D. A. 2005, ApJ, 624, 606

Johnson, R. E., Markevitch, M., Wegner, G. A., Jones, C., \& Forman, W. R. 2010, ApJ, 710, 1776

Jones, C., \& Forman, W. 1992, in Clusters and Superclusters of Galaxies, ed. A. C. Fabian, NATO ASIC Proc. 366, 49

Jones, C., \& Forman, W. 1999, ApJ, 511, 65

Kapferer, W., Ferrari, C., Domainko, W., et al. 2006, A\&A, 447, 827

Kempner, J. C., Sarazin, C. L., \& Ricker, P. M. 2002, ApJ, 579, 236

Lorenz, M. O. 1905, Publications of the American Statistical Association, 9, 209

Lotz, J. M., Primack, J., \& Madau, P. 2004, AJ, 128, 163

Maughan, B. J., Jones, C., Forman, W., \& Van Speybroeck, L. 2008, ApJS, 174, 117

Maurogordato, S., Sauvageot, J. L., Bourdin, H., et al. 2011, A\&A, 525, A79

Mohr, J. J., Fabricant, D. G., \& Geller, M. J. 1993, ApJ, 413, 492

Murgia, M., Govoni, F., Feretti, L., \& Giovannini, G. 2010, A\&A, 509, A86

Neumann, D. M., Lumb, D. H., Pratt, G. W., \& Briel, U. G. 2003, A\&A, 400, 811

Nurgaliev, D., McDonald, M., Benson, B. A., et al. 2013, ApJ, 779, 112

Okabe, N., Zhang, Y.-Y., Finoguenov, A., et al. 2010, ApJ, 721, 875

Poole, G. B., Fardal, M. A., Babul, A., et al. 2006, MNRAS, 373, 881

Rasia, E., Meneghetti, M., \& Ettori, S. 2013, ARA\&A, 8, 40

Reiprich, T. H., Sarazin, C. L., Kempner, J. C., \& Tittley, E. 2004, ApJ, 608, 179

Ritchie, B. W., \& Thomas, P. A. 2002, MNRAS, 329, 675

Rossetti, M., Ghizzardi, S., Molendi, S., \& Finoguenov, A. 2007, A\&A, 463, 839

Santos, J. S., Rosati, P., Tozzi, P., et al. 2008, A\&A, 483, 35

Scarlata, C., Carollo, C. M., Lilly, S., et al. 2007, ApJS, 172, 406

Schuecker, P. 2005, in Rev. Mod. Astron. 18, ed. S. Röser, 76

Schuecker, P., Böhringer, H., Reiprich, T. H., \& Feretti, L. 2001, A\&A, 378, 408

Tozzi, P., \& Norman, C. 2001, ApJ, 546, 63

Venturi, T. 2011, Mem. Soc. Astron. It., 82, 499

Venturi, T., Bardelli, S., Dallacasa, D., et al. 2003, A\&A, 402, 913

Vikhlinin, A., Burenin, R., Forman, W. R., et al. 2007, in Heating versus

Cooling in Galaxies and Clusters of Galaxies, eds. H. Böhringer, G. W. Pratt, A. Finoguenov, \& P. Schuecker, 48

Vikhlinin, A., Burenin, R. A., Ebeling, H., et al. 2009, ApJ, 692, 1033 (V09)

Wang, T., Huang, J.-S., Faber, S. M., et al. 2012, ApJ, 752, 134

Weißmann, A., Böhringer, H., Suhada, R., \& Ameglio, S. 2013, A\&A, 549, A19

Yitzhaki, S. 1991, J. Business Economic Statistics, 9, 235

Zamojski, M. A., Schiminovich, D., Rich, R. M., et al. 2007, ApJS, 172, 468

Zhang, Y.-Y., Okabe, N., Finoguenov, A., et al. 2010, ApJ, 711, 1033

Ziparo, F., Braglia, F. G., Pierini, D., et al. 2012, MNRAS, 420, 2480 


\section{Appendix A}

Table A.1. Morphology parameters value of relaxed clusters (V09).

\begin{tabular}{|c|c|c|c|c|c|c|c|}
\hline Cluster name & Gini & $M_{20}$ & Concentration & Asymmetry & Smoothness & $G_{M}$ & Ellipticity \\
\hline 571 & $357 \pm 0.002$ & $-1.492 \pm 0.217$ & $370 \pm 0.091$ & $0.293 \pm 0.232$ & $0.284 \pm 0.004$ & $0.259 \pm 0.001$ & $0.084 \pm 0.043$ \\
\hline A2199 & $0.612 \pm 0.001$ & $-1.897 \pm 0.188$ & $1.165 \pm 0.097$ & $0.502 \pm 0.078$ & $0.329 \pm 0.005$ & $0.378 \pm 0.002$ & $0.083 \pm 0.037$ \\
\hline 2A 0335 & $0.666 \pm 0.001$ & $-2.123 \pm 0.258$ & $1.817 \pm 0.237$ & $0.341 \pm 0.128$ & $0.321 \pm 0.008$ & $0.209 \pm 0.001$ & $0.082 \pm 0.041$ \\
\hline A496 & $0.564 \pm 0.002$ & $-1.667 \pm 0.320$ & $1.227 \pm 0.086$ & $0.577 \pm 0.081$ & $0.205 \pm 0.005$ & $0.284 \pm 0.001$ & $0.063 \pm 0.036$ \\
\hline A3667 & $0.435 \pm 0.001$ & $-1.590 \pm$ & $0.918 \pm$ & $0.302 \pm 0.132$ & $0.536 \pm 0.002$ & $0.285=$ & 0.079 \\
\hline A754 & $0.341 \pm 0.001$ & $-1.053 \pm 0.076$ & $0.798 \pm 0.052$ & $0.406 \pm 0.058$ & $0.524 \pm 0.003$ & $0.308 \pm 0.001$ & $0.202 \pm 0.006$ \\
\hline A85 & $0.624 \pm 0.001$ & $-1.779 \pm 0.260$ & $1.447 \pm 0.067$ & $0.633 \pm 0.117$ & $0.464 \pm 0.003$ & $0.279 \pm 0.001$ & $0.026 \pm 0.006$ \\
\hline A2029 & $0.718 \pm 0.002$ & $-2.088 \pm 0.315$ & $1.603 \pm 0.192$ & $0.445 \pm 0.165$ & $0.163 \pm 0.009$ & $0.280 \pm 0.001$ & $0.113 \pm 0.023$ \\
\hline A478 & $0.703 \pm 0.003$ & $-2.145 \pm 0.255$ & $1.613 \pm 0.162$ & $0.286 \pm 0.165$ & $0.248 \pm 0.008$ & $0.279 \pm 0.001$ & $0.137 \pm 0.062$ \\
\hline A1795 & $0.779 \pm 0.002$ & $-2.148 \pm 0.296$ & 0.097 & $0.351 \pm 0.053$ & $0.143 \pm 0.012$ & $0.389 \pm 0.001$ & $0.107 \pm 0.029$ \\
\hline A3558 & $0.327 \pm 0.001$ & $-1.448 \pm 0.226$ & $0.866 \pm 0.087$ & $0.358 \pm 0.153$ & $0.520 \pm 0.002$ & $0.276 \pm 0.003$ & $0.117 \pm 0.068$ \\
\hline A 2142 & $0.564 \pm 0.002$ & $-1.959 \pm 0.218$ & $1.322 \pm 0.199$ & $0.365 \pm 0.263$ & $0.329 \pm 0.003$ & $0.239 \pm 0.000$ & $0.174 \pm 0.044$ \\
\hline A2256 & $0.373 \pm 0.000$ & $-1.273 \pm 0.064$ & $0.896 \pm 0.047$ & $0.333 \pm 0.119$ & $1.024 \pm 0.002$ & $0.277 \pm 0.000$ & $0.133 \pm 0.041$ \\
\hline A4038 & $0.503 \pm 0.002$ & $-1.929 \pm 0.193$ & $1.291 \pm 0.181$ & $0.297 \pm 0.191$ & $0.563 \pm 0.001$ & $0.206 \pm 0.000$ & $0.140 \pm 0.038$ \\
\hline A2147 & $0.268 \pm 0.001$ & $-1.243 \pm 0.102$ & $0.753 \pm 0.178$ & $0.359 \pm 0.150$ & $1.188 \pm 0.002$ & $0.305 \pm 0.000$ & $0.083 \pm 0.057$ \\
\hline A3266 & $0.220 \pm 0.001$ & $-1.196 \pm 0.232$ & & $0.276 \pm 0.256$ & $0.514 \pm 0.005$ & $0.279 \pm 0.002$ & $0.103 \pm 0.069$ \\
\hline & $0.439 \pm 0.000$ & $-1.734 \pm 0.121$ & & $0.222 \pm 0.207$ & $0.739 \pm 0.002$ & $0.205 \pm 0.000$ & $2 \pm 0.040$ \\
\hline A2052 & $0.622 \pm$ & 37 & 95 & 0.6 & 0.003 & $0.332 \pm$ & .046 \\
\hline Hydra-A & $0.543 \pm 0.004$ & $-2.087 \pm 0.383$ & \pm 0.248 & 0.2 & 0.152 & $0.149 \pm 0.002$ & .065 \\
\hline & $417 \pm 0.000$ & $-1.158 \pm 0.066$ & .085 & 0.5 & .001 & $0.434 \pm$ & 057 \\
\hline & $6 \pm$ & $-1.729 \pm 0.169$ & .156 & 0.4 & .001 & $0.281 \pm$ & 057 \\
\hline $\mathrm{A} 1$ & $2 \pm$ & -1.8 & 86 & 0.3 & 003 & $0.273 \pm$ & .067 \\
\hline A3158 & $0.470 \pm 0.001$ & $-1.767 \pm 0.104$ & 0.138 & $0.267 \pm 0$ & 0.001 & $0.232 \pm 0.000$ & 0.043 \\
\hline MKW3s & $0.509 \pm 0.003$ & $-1.988 \pm 0.273$ & $1.287 \pm 0.193$ & $0.222 \pm 0.338$ & $0.317 \pm 0.006$ & $0.173 \pm 0.001$ & \pm 0.056 \\
\hline A1736 & $0.215 \pm 0.001$ & $-0.932 \pm 0.101$ & $0.613 \pm 0.208$ & $0.415 \pm 0.109$ & $1.379 \pm 0.001$ & $0.352 \pm 0.001$ & 0.080 \\
\hline EXO0422 & $0.622 \pm 0.002$ & $-2.304 \pm 0.228$ & $1.630 \pm 0.268$ & $0.355 \pm 0.183$ & $0.911 \pm 0.005$ & $0.254 \pm 0.000$ & 0.071 \\
\hline A4059 & $0.424 \pm 0.002$ & $-1.747 \pm 0.267$ & $1.110 \pm 0.107$ & $0.211 \pm 0.170$ & $0.135 \pm 0.005$ & $0.167 \pm 0.001$ & $0.093 \pm 0.044$ \\
\hline 395 & $0.394 \pm 0.001$ & $-0.801 \pm 0.098$ & $0.593 \pm 0.093$ & $0.734 \pm 0.071$ & $1.411 \pm 0.001$ & $0.473 \pm 0.001$ & $0.228 \pm 0.090$ \\
\hline A2589 & $0.381 \pm 0.001$ & $-1.415 \pm 0.230$ & $0.862 \pm 0.166$ & $0.471 \pm 0.055$ & $0.669 \pm 0.003$ & $0.317 \pm 0.001$ & $0.045 \pm 0.037$ \\
\hline A 3112 & $0.544 \pm 0.004$ & $-2.121 \pm 0.406$ & $1.420 \pm 0.204$ & $0.276 \pm 0.340$ & $0.309 \pm 0.013$ & $0.170 \pm 0.004$ & $0.143 \pm 0.072$ \\
\hline 562 & $0.541 \pm 0.001$ & $-1.870 \pm 0.099$ & $1.204 \pm 0.175$ & $0.542 \pm 0.019$ & $1.211 \pm 0.001$ & $0.384 \pm 0.000$ & $0.107 \pm 0.051$ \\
\hline A1651 & $0.569 \pm 0.001$ & $-1.957 \pm 0.207$ & $1.321 \pm 0.180$ & $0.354 \pm($ & $1.001 \pm 0.002$ & $0.266 \pm 0.000$ & 0.049 \\
\hline & $9 \pm$ & $9 \pm 0.178$ & 1. & 0. & 0.6 & $0.229 \pm$ & 079 \\
\hline A2204 & $0.732 \pm$ & $-2.611 \pm$ & 2.0 & $0.361 \pm$ & 0.54 & $0.208 \pm$ & .062 \\
\hline A576 & $0.308 \pm$ & $-1.651 \pm$ & 147 & $0.267 \pm$ & 002 & $0.251 \pm$ & .068 \\
\hline A2657 & $0.380 \pm 0.001$ & $-1.607 \pm 0.194$ & $0.998 \pm 0.222$ & $0.402 \pm 0.157$ & $1.034 \pm 0.003$ & $0.250 \pm 0.001$ & \pm 0.051 \\
\hline A3391 & $0.408 \pm 0.001$ & $-1.549 \pm 0.079$ & $0.952 \pm 0.123$ & $0.431 \pm 0.096$ & $1.335 \pm 0.001$ & $0.332 \pm 0.000$ & $0.115 \pm 0.051$ \\
\hline A2065 & $0.477 \pm 0.001$ & $-1.697 \pm 0.166$ & $1.218 \pm 0.091$ & $0.402 \pm 0.155$ & $0.549 \pm 0.003$ & $0.230 \pm 0.001$ & $0.149 \pm 0.049$ \\
\hline A 1650 & $0.673 \pm 0.001$ & $-1.869 \pm 0.318$ & $1.371 \pm 0.165$ & $0.429 \pm 0.087$ & $0.476 \pm 0.004$ & $0.379 \pm 0.002$ & $0.122 \pm 0.093$ \\
\hline A 3822 & $0.450 \pm 0.001$ & $-1.542 \pm 0.146$ & $1.045 \pm 0.197$ & $0.536 \pm 0.071$ & $1.450 \pm 0.002$ & $0.370 \pm 0.000$ & $0.119 \pm 0.066$ \\
\hline S 1101 & $0.645 \pm 0.002$ & $-2.111 \pm 0.214$ & $1.561 \pm 0.241$ & $0.238 \pm 0.199$ & $0.461 \pm 0.011$ & $0.172 \pm 0.002$ & $0.110 \pm 0.062$ \\
\hline A2163 & $0.378 \pm 0.002$ & $-1.443 \pm 0.179$ & $0.943 \pm 0.168$ & $0.281 \pm 0.158$ & $0.272 \pm 0.004$ & $0.196 \pm 0.002$ & $0.082 \pm 0.061$ \\
\hline ZwCl1215 & $0.439 \pm 0.001$ & $-1.625 \pm 0.109$ & $1.040 \pm 0.103$ & $0.369 \pm 0.117$ & $1.204 \pm 0.002$ & $0.294 \pm 0.000$ & $0.120 \pm 0.086$ \\
\hline RXJ1504 & $0.807 \pm 0.004$ & $-2.471 \pm 0.400$ & $2.128 \pm 0.322$ & $0.397 \pm 0.188$ & $0.370 \pm 0.038$ & $0.242 \pm 0.003$ & $0.124 \pm 0.067$ \\
\hline A2597 & $0.781 \pm 0.003$ & $-2.426 \pm 0.558$ & $2.277 \pm 0.389$ & $0.397 \pm 0.266$ & $0.295 \pm 0.010$ & $0.277 \pm 0.001$ & $0.152 \pm 0.077$ \\
\hline A133 & $0.508 \pm 0.003$ & $-2.201 \pm 0.396$ & $1.438 \pm 0.301$ & $0.254 \pm 0.292$ & $0.320 \pm 0.008$ & $0.179 \pm 0.002$ & $0.094 \pm 0.079$ \\
\hline A2244 & $0.638 \pm 0.001$ & $-1.983 \pm 0.287$ & $1.445 \pm 0.232$ & $0.266 \pm 0.151$ & $0.314 \pm 0.003$ & $0.219 \pm 0.005$ & $0.030 \pm 0.059$ \\
\hline A3376 & $0.413 \pm 0.000$ & $-1.183 \pm 0.063$ & $0.803 \pm 0.153$ & $0.564 \pm 0.045$ & $1.159 \pm 0.001$ & $0.397 \pm 0.001$ & $0.072 \pm 0.0869$ \\
\hline
\end{tabular}

Notes. Values are listed with appropriate uncertainties of $1 \sigma$ for each parameter. 
Table A.2. Morphology parameters value of (V09) non-relaxed clusters.

\begin{tabular}{|c|c|c|c|c|c|c|c|}
\hline Cluster name & Gini & $M_{20}$ & Concentration & Asymmetry & Smoothness & $G_{M}$ & Ellipticity \\
\hline $0302-0423$ & $.796 \pm 0.010$ & $-2.511 \pm 0.750$ & $2.186 \pm 0.769$ & $0.322 \pm 0.281$ & $0.767 \pm 0.027$ & $0.345 \pm 0.006$ & $0.071 \pm 0.231$ \\
\hline $1212+2733$ & $.417 \pm 0.004$ & $-1.670 \pm 0.349$ & $0.993 \pm 0.553$ & $0.388 \pm 0.377$ & $1.206 \pm 0.003$ & $0.293 \pm 0.003$ & $0.102 \pm 0.212$ \\
\hline 0350-3801 & $0.465 \pm 0.003$ & $-1.734 \pm 0.538$ & $1.117 \pm 1.046$ & $0.591 \pm 0.288$ & $1.453 \pm 0.000$ & $0.386 \pm 0.001$ & $0.082=$ \\
\hline 0318-0302 & $0.554 \pm 0.003$ & $-1.631 \pm 0.675$ & $1.197 \pm 0.900$ & $0.532 \pm 0.474$ & $1.306 \pm 0.002$ & $0.339 \pm 0.003$ & $0.073 \pm 0.000$ \\
\hline $0159+0030$ & $0.559 \pm 0.008$ & $-2.288 \pm 1.139$ & $1.531 \pm 0.628$ & $0.597 \pm 0.400$ & $1.198 \pm 0.002$ & $0.334 \pm 0.002$ & $0.022 \pm 0.000$ \\
\hline $0958+4702$ & $0.550 \pm 0.007$ & $-2.165 \pm 0.557$ & $1.442 \pm 1.034$ & $0.416 \pm 0.354$ & $1.310 \pm 0.000$ & $0.354 \pm 0.001$ & $0.015 \pm 0.000$ \\
\hline $0809+2811$ & $366 \pm 0.003$ & $-1.334 \pm 0.738$ & $0.914 \pm 1.160$ & $0.449 \pm 0.370$ & $1.291 \pm 0.002$ & $0.309 \pm 0.003$ & $0.115 \pm 0.307$ \\
\hline $1416+4446$ & $528 \pm 0.010$ & $-1.972 \pm 0.466$ & $1.442 \pm 1.005$ & $0.553 \pm 0.472$ & $1.009 \pm 0.013$ & $0.274 \pm 0.003$ & $0.103 \pm 0.000$ \\
\hline $1312+3900$ & $322 \pm 0.002$ & $-1.359 \pm 0.279$ & $0.837 \pm 0.612$ & $0.507 \pm 0.453$ & $1.443 \pm 0.000$ & $0.354 \pm 0.001$ & $0.030=$ \\
\hline $1003+3253$ & $0.595 \pm 0.008$ & $-2.369 \pm 0.779$ & $1.748 \pm 1.035$ & $0.451 \pm 0.259$ & $1.166 \pm 0.000$ & $0.353 \pm 0.002$ & 0.100 \\
\hline & $0.347 \pm 0.003$ & $-0.961 \pm 0.170$ & $0.759 \pm 0.293$ & $0.623 \pm 0.303$ & $1.591 \pm 0.000$ & $0.432 \pm 0.002$ & .000 \\
\hline 170 & $0.479 \pm 0.008$ & $-2.017 \pm 0.817$ & & & .005 & $0.240 \pm$ & 000 \\
\hline 164 & $0.481 \pm$ & $-1.913 \pm 0.483$ & 03 & & 000 & $0.282 \pm$ & 000 \\
\hline $0522-3624$ & $0.444 \pm 0.008$ & $-1.429 \pm 0.356$ & $6 \pm 1.353$ & $0.471 \pm$ & 003 & $0.307 \pm$ & 0.087 \\
\hline $1222+2709$ & $444 \pm 0.005$ & $-1.983 \pm 0.241$ & 135 & $0.412 \pm$ & .000 & $0.301 \pm 0.001$ & .000 \\
\hline 741 & $197 \pm 0.007$ & $-2.031 \pm 0.239$ & 198 & $0.429=$ & 000 & $0.303 \pm$ & .000 \\
\hline $0853+5759$ & $0.418 \pm 0.004$ & $-1.294 \pm 0.432$ & $1.034 \pm 0.746$ & $0.715 \pm 0.361$ & $1.075 \pm 0.000$ & $0.331 \pm 0.004$ & 0.030 \\
\hline $0333-2456$ & $414 \pm 0.002$ & $-1.435 \pm 0.218$ & $0.947 \pm 0.199$ & $0.462 \pm 0.315$ & $1.363 \pm 0.000$ & $0.341 \pm 0.001$ & $0.117 \pm 0.000$ \\
\hline $0926+1242$ & $506 \pm 0.003$ & $-1.764 \pm 0.584$ & $1.212 \pm 1.019$ & $0.558 \pm 0.336$ & $1.420 \pm 0.000$ & $0.399 \pm 0.002$ & $0.158 \pm 0.000$ \\
\hline $0030+2618$ & $0.258 \pm 0.004$ & $-1.639 \pm 0.336$ & $0.833 \pm 0.392$ & $0.258 \pm 0.502$ & $0.578 \pm 0.006$ & $0.282 \pm 0.002$ & $0.045 \pm 0.130$ \\
\hline $1002+6858$ & $474 \pm 0.005$ & $-1.914 \pm 0.625$ & $1.243 \pm 0.861$ & $0.475 \pm 0.217$ & $1.388 \pm 0.000$ & $0.362 \pm 0.001$ & $0.065 \pm 0.000$ \\
\hline $1524+0957$ & $343 \pm 0.003$ & $-1.343 \pm 0.380$ & $0.918 \pm 0.429$ & $0.366 \pm 0.495$ & $1.065 \pm 0.000$ & $0.262 \pm 0.005$ & $0.102 \pm 0.000$ \\
\hline $1357+6232$ & $419 \pm 0.004$ & $-1.696 \pm 0.400$ & $1.045 \pm 1.131$ & $0.385 \pm 0.394$ & $0.945 \pm 0.015$ & $0.262 \pm 0.003$ & $0.019=$ \\
\hline $1354-($ & .003 & $-1.481 \pm 0.344$ & \pm 0.756 & $0.477 \pm 0.333$ & $0.977 \pm 0.009$ & $0.308 \pm 0.001$ & $0.028 \pm 0.000$ \\
\hline $1120+2326$ & $0.367 \pm 0.002$ & $-1.237 \pm 0.424$ & $0.842 \pm 0.323$ & $0.439 \pm 0.448$ & $0.964 \pm 0.000$ & $0.272 \pm 0.002$ & 0.000 \\
\hline $0956+4107$ & $0.416 \pm 0.002$ & $-1.450 \pm 0.251$ & $1.010 \pm 0.107$ & $0.388 \pm 0.365$ & $1.156 \pm 0.000$ & $0.300 \pm 0.002$ & $0.147 \pm 0.000$ \\
\hline $0328-2140$ & $0.430 \pm$ & $-1.767 \pm 0.689$ & $1.068 \pm 1.208$ & $0.480 \pm$ & $0.944 \pm 0.000$ & $0.281 \pm 0.003$ & $0.073 \pm 0.000$ \\
\hline $1120+4318$ & $0.506 \pm 0.006$ & $-1.665 \pm 0.428$ & $1.201 \pm 0.851$ & $0.506 \pm 0$ & $1.149 \pm 0.000$ & $0.287 \pm 0.006$ & 0.06 \\
\hline $1334+5031$ & $0.428 \pm 0.005$ & $-1.815 \pm 0.319$ & $1.045 \pm$ & 0.517 & 000 & $0.376 \pm 0.001$ & 0.068 \\
\hline $0542-4100$ & $0.389 \pm 0.005$ & $-1.508 \pm 0.429$ & $1.034 \pm 0.332$ & $0.464 \pm 0.499$ & $0.935 \pm 0.000$ & $0.264 \pm 0.004$ & $0.099 \pm$ \\
\hline $1202+5751$ & $0.303 \pm 0.002$ & $-1.231 \pm 0.326$ & $0.807 \pm 0.113$ & $0.409 \pm 0.440$ & $1.191 \pm 0.000$ & $0.297 \pm 0.002$ & 0.106 \\
\hline $0405-4100$ & $0.489 \pm 0.004$ & $-1.976 \pm 0.381$ & $1.346 \pm 0.845$ & $0.448 \pm 0.369$ & $0.903 \pm 0.000$ & $0.291 \pm 0.002$ & $0.014 \pm 0.000$ \\
\hline $1221+4918$ & $0.362 \pm 0.002$ & $-1.488 \pm 0.550$ & $0.971 \pm 1.002$ & $0.282 \pm 0.444$ & $0.811 \pm 0.000$ & $0.230 \pm 0.003$ & $0.091 \pm 0.000$ \\
\hline $0230+1836$ & $0.310 \pm 0.002$ & $-1.079 \pm 0.588$ & $0.772 \pm 0.158$ & $0.442 \pm 0.459$ & $1.093 \pm 0.000$ & $0.296 \pm 0.002$ & $0.130 \pm 0.000$ \\
\hline & $0.337 \pm 0.003$ & $-1.532 \pm 0.268$ & $0.818 \pm 0.107$ & $0.510 \pm 0.378$ & $1.183 \pm 0.000$ & $0.348 \pm 0.003$ & $0.169 \pm 0.000$ \\
\hline $1226+3332$ & $0.585 \pm 0.006$ & $-1.859 \pm 0.929$ & $1.435 \pm 0.799$ & $0.277 \pm 0.151$ & $0.411 \pm 0.018$ & $0.168 \pm 0.007$ & $0.035 \pm 0.246$ \\
\hline
\end{tabular}

Notes. Values are listed with appropriate uncertainties of $1 \sigma$ for each parameter.

Table A.3. Three morphology parameters value of radio halo clusters, except for the V09 radio halo clusters (A754, A2256, A401, A3562, A399, and A2163).

\begin{tabular}{cccc}
\hline \hline Cluster name & Gini & $M_{20}$ & Concentration \\
\hline A1914 & $0.621 \pm 0.002$ & $-1.567 \pm 0.247$ & $1.282 \pm 0.266$ \\
A2218 & $0.472 \pm 0.001$ & $-1.720 \pm 0.264$ & $1.122 \pm 0.156$ \\
A665 & $0.427 \pm 0.002$ & $-1.320 \pm 0.269$ & $1.09 \pm 0.229$ \\
A520 & $0.357 \pm 0.001$ & $-1.090 \pm 0.154$ & $0.814 \pm 0.322$ \\
A773 & $0.493 \pm 0.003$ & $-1.697 \pm 0.249$ & $1.215 \pm 0.255$ \\
IE 0657-56 & $0.358 \pm 0.003$ & $-1.073 \pm 0.195$ & $0.783 \pm 0.156$ \\
A2255 & $0.270 \pm 0.000$ & $-1.441 \pm 0.110$ & $0.798 \pm 0.126$ \\
A2319 & $0.420 \pm 0.001$ & $-1.491 \pm 0.141$ & $1.041 \pm 0.088$ \\
A1300 & $0.477 \pm 0.004$ & $-1.516 \pm 0.412$ & $1.114 \pm 0.335$ \\
A1758 & $0.371 \pm 0.002$ & $-0.883 \pm 0.184$ & $0.741 \pm 0.414$ \\
A1995 & $0.520 \pm 0.002$ & $-1.807 \pm 0.286$ & $1.172 \pm 0.405$ \\
A2034 & $0.420 \pm 0.001$ & $-1.645 \pm 0.149$ & $0.930 \pm 0.101$ \\
A209 & $0.449 \pm 0.002$ & $-1.754 \pm 0.248$ & $1.110 \pm 0.208$ \\
A2219 & $0.407 \pm 0.002$ & $-1.642 \pm 0.254$ & $0.986 \pm 0.125$ \\
A2294 & $0.463 \pm 0.002$ & $-1.681 \pm 0.305$ & $1.172 \pm 0.420$ \\
A2744 & $0.300 \pm 0.002$ & $-1.370 \pm 0.222$ & $0.821 \pm 0.271$ \\
A521 & $0.231 \pm 0.002$ & $-1.055 \pm 0.176$ & $0.670 \pm 0.417$ \\
A697 & $0.382 \pm 0.002$ & $-1.720 \pm 0.265$ & $1.07 \pm 0.186$ \\
RXC J2003.5-2323 & $0.240 \pm 0.012$ & $-1.023 \pm 0.223$ & $0.730 \pm 0.267$ \\
\hline
\end{tabular}

Notes. Values are listed with appropriate uncertainties of $1 \sigma$ for each parameter. 
A\&A 575, A127 (2015)

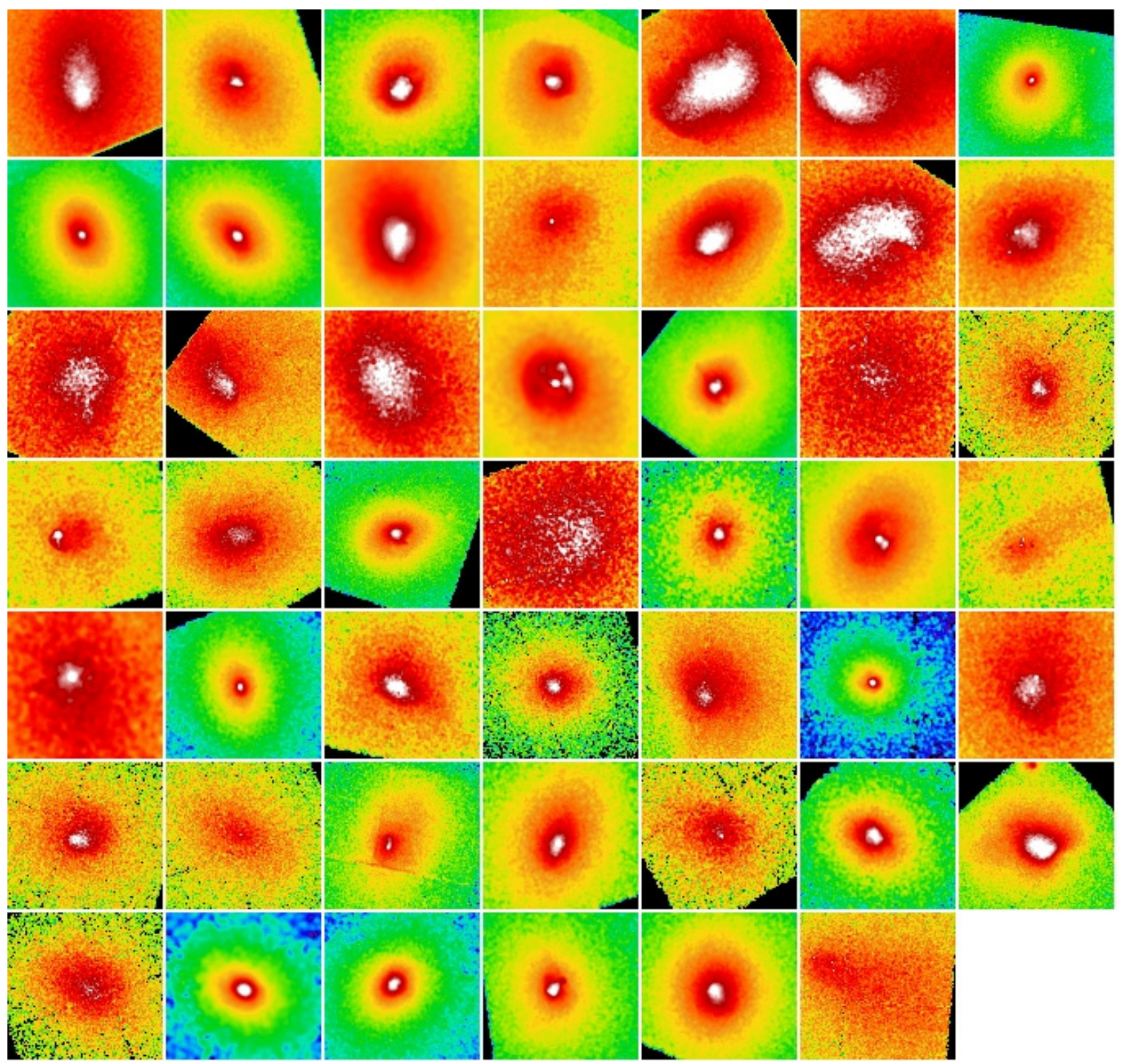

Fig. A.1. Low- $z$ clusters of the V09. Cluster names of panels from top left to bottom right are listed as in Table 1. Each image has the same colour, scale $(\log )$, and length. 
V. Parekh et al.: Morphology parameters: substructure identification in X-ray galaxy clusters

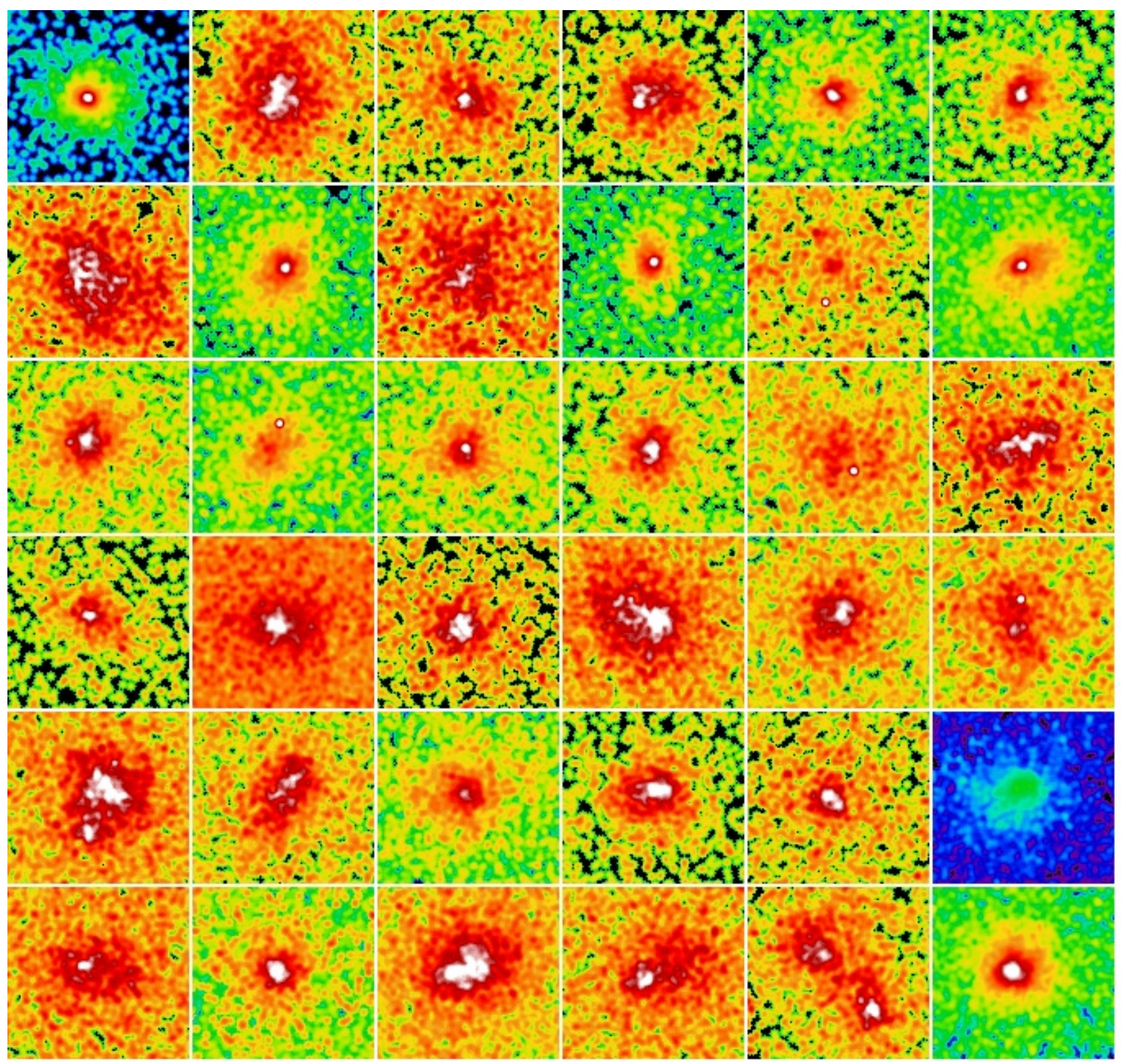

Fig. A.2. High- $z$ clusters of the V09. Cluster names of panels from top left to bottom right are listed as in Table 2. Each image has the same colour, scale $(\log )$, and length. 


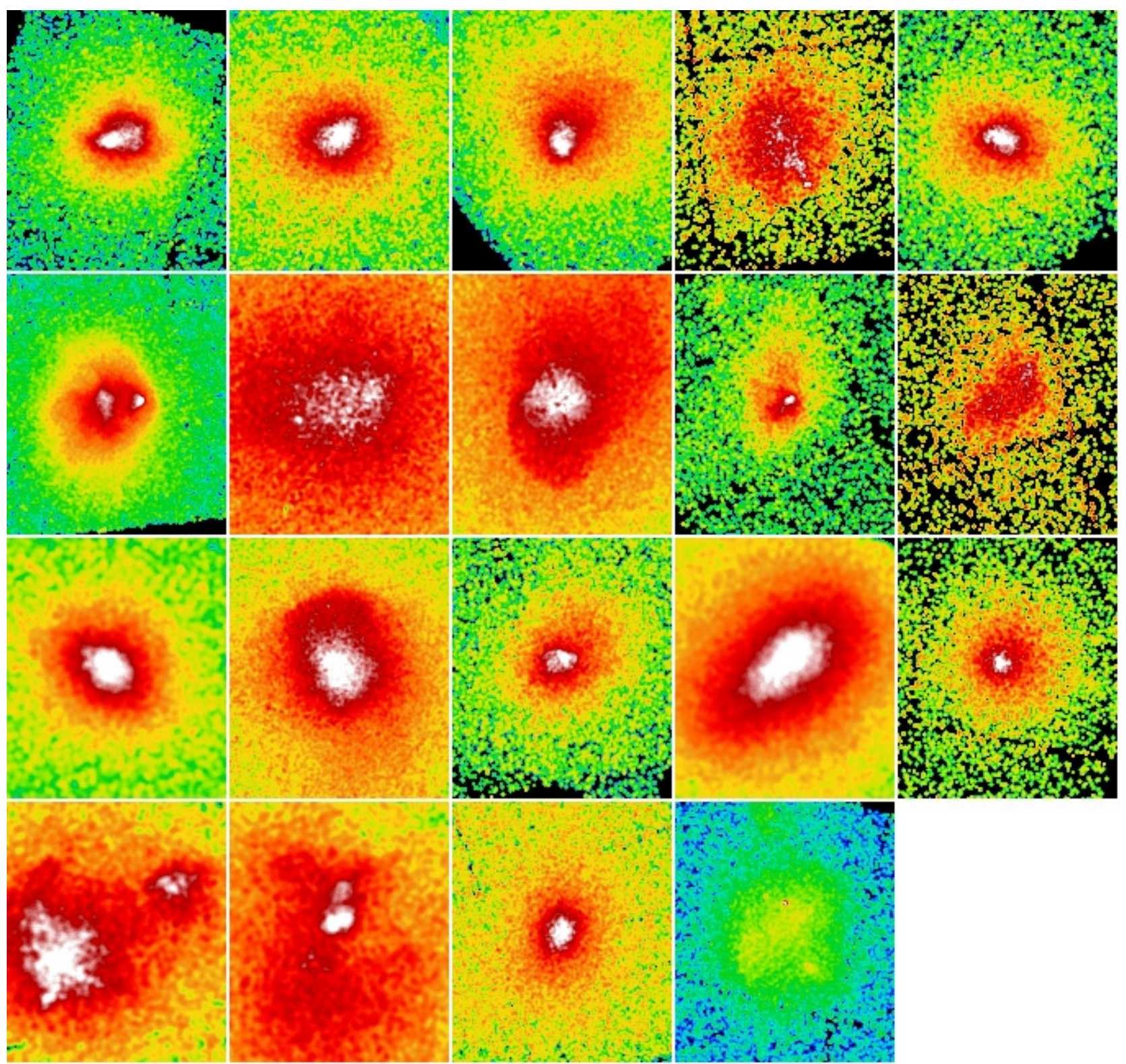

Fig. A.3. Radio halo clusters (Giovannini et al. 2009). Cluster names of panels from top left to bottom right are listed as in Table 9, except for the V09 radio halo clusters (A754, A2256, A401, A3562, A399, and A2163). Each image has the same colour, scale (log), and length. 